To appear in ApJ Supplement

Preprint typeset using IATEX style emulateapj v. September 4, 2018

\title{
CHAMPLANE OPTICAL SURVEY: MOSAIC PHOTOMETRY
}

\author{
Ping ZhaO ${ }^{1,2, *}$, Jonathan E. Grindlay, Jae Sub Hong, Silas Laycock ${ }^{1,2}$, Xavier P. Koenig ${ }^{1}$, Eric M. Schlegel, \\ AND MAUREEN VAN DEN BERG ${ }^{2}$ \\ Harvard-Smithsonian Center for Astrophysics, 60 Garden Street, Cambridge, MA 02138 USA \\ To appear in ApJ Supplement
}

\begin{abstract}
The ChaMPlane survey to identify and analyze the serendipitous X-ray sources in deep Galactic plane fields incorporates the ChaMPlane Optical Survey, which is one of NOAO's Long-term Survey Programs. We started this optical imaging survey in March 2000 and completed it in June 2005. Using the NOAO 4-m telescopes with the Mosaic cameras at CTIO and KPNO, deep images of the ChaMPlane fields are obtained in V, R, I and $\mathrm{H} \alpha$ bands. This paper describes the process of observation, data reduction and analysis of fields included in the ChaMPlane Optical Survey, and describes the search for $\mathrm{H} \alpha$ emission objects and Chandra optical counterparts. We illustrate these procedures using the ChaMPlane field for the black hole X-ray binary GRO J0422+32 as an example. Subject headings: Chandra, X-ray, ChaMPlane, Galactic plane, survey
\end{abstract}

\section{INTRODUCTION}

The ChaMPlane Survey ${ }^{3}$ identifies serendipitous Xray sources located to arcsec precision in the Galactic plane fields from the Chandra archive, in order to determine the populations of accretion-powered binaries in the Galaxy (Grindlav et al. (2005), and see Grindlav et al. (2003); Zhao et al. (2003) for early descriptions).

The primary goals of this survey are: 1) to identify Cataclysmic Variables (CVs) and quiescent Low Mass Xray Binaries (qLMXBs: which contain either black hole or neutron star primaries) in order to measure their number and space density luminosity functions; and 2) to determine the distributions of High Mass X-ray Binaries with Be star secondaries. The secondary goal is to study the distributions of stellar coronal source and diffuse Xray objects in the Galactic Plane. See Grindlav et al. (2005) for a complete description of the survey goals, selection criteria and initial results.

ChaMPlane consists of an X-ray and an Optical survey. The X-ray survey, supported by NASA through Chandra archival proposals, searches for X-ray sources detected serendipitously in Chandra archival fields. In the Optical Survey, supported by NOAO, we look for $\mathrm{H} \alpha$ emission sources and the Chandra optical counterparts in 4-meter telescope Mosaic images taken at CTIO and KPNO. We successfully conducted the ChaMPlane Optical Survey from March 2000 to June 2005. This 5 year survey produced 65 Mosaic fields covering about 23 square degrees and 154 ACIS observations on 105 distinct Chandra fields (defined as groups of individual observations whose aimpoints are $\geqslant 4^{\prime}$ apart) in the Galactic

\footnotetext{
* Send request to Ping Zhao at zhao@cfa.harvard.edu

${ }^{1}$ Visiting Astronomer, Cerro Tololo Inter-American Observatory. National Optical Astronomy Observatory, which is operated by the Association of Universities for Research in Astronomy, Inc. (AURA) under cooperative agreement with the National Science Foundation.

${ }^{2}$ Visiting Astronomer, Kitt Peak National Observatory, National Optical Astronomy Observatory, which is operated by the Association of Universities for Research in Astronomy, Inc. (AURA) under cooperative agreement with the National Science Foundation.

${ }^{3}$ http://hea-www.harvard.edu/ChaMPlane
}

plane during Chandra Cycles 1-6.

In this paper we describe the methods of the ChaMPlane Optical Survey and the procedures to search for $\mathrm{H} \alpha$ emission objects and Chandra optical counterparts. Section 2 describes the observational approach. Section 3 describes the Mosaic data reduction. Section 4 describes the photometric analysis and calibration. Section 5 describes the photometric results - the optical catalog. Section 6 gives the astrometric accuracy of the catalog. Sections 7 and 8 describe the search for $\mathrm{H} \alpha$ emission objects and Chandra optical counterparts, respectively. Section 9 describes the data product, and uses the results from the GRO J0422+32 field as an example.

\section{OPTICAL IMAGING}

Under the NOAO Long-term Survey Program, we were granted 5 nights CTIO 4-m and 1-2 nights KPNO 4$\mathrm{m}$ telescope time each year for 5 years. Before this long term program officially started in December 2000 at KPNO, we conducted a pilot run using the CTIO 4-m Mosaic on March 13-15, 2000. Under this Survey Program, we are committed to establish an archival database to provide the community with all of our optical images as well as a photometrically-calibrated star catalog.

ChaMPlane fields are selected from the Chandra target list based on criteria given in Grindlav et al. (2005). Deep optical imaging is the crucial first step of the ChaMPlane survey. It serves two purposes: 1) to identify candidate optical counterparts of the Chandra sources and to measure their optical magnitudes and $F_{X} / F_{\text {opt }}$ ratios for approximate spectral classification and constraints on reddening; and 2) to identify CVs and qLMXBs by their ubiquitous $\mathrm{H} \alpha$ excess as "blue" objects in the $\mathrm{R}$ vs. $(\mathrm{H} \alpha-\mathrm{R})$ diagram. It also paves the way for the next step - spectroscopic follow-up for classification on identified ChaMPlane objects.

\subsection{Instrument}

The images were taken with the Mosaic-I (KPNO) and Mosaic-II (CTIO) cameras ${ }^{4}$. The Mosaic camera is a

\footnotetext{
4 The complete instrument information can be found at http://www.noao.edu/noao/mosaic/
} 
TABLE 1

Champlane Observations: Mosaic Imaging

\begin{tabular}{|c|c|c|c|}
\hline$\overline{\text { Code }}$ & Telescope & Observing Date & Fields \\
\hline ctio00 & CTIO 4-m & Mar. $13-15,2000$ & 9 \\
\hline kpno00 & KPNO 4-m & Dec. $5-6,2000$ & 4 \\
\hline ctio01 & CTIO 4-m & May $13-17,2001$ & 8 \\
\hline kpno01 & KPNO 4-m & Oct. 25,2001 & 3 \\
\hline kpno02 & KPNO 4-m & Dec. $7-8,2002$ & 6 \\
\hline ctio03 & CTIO 4-m & Jun. $2-6,2003$ & 11 \\
\hline kpno03 & KPNO 4-m & Jan. $30-31,2004$ & 2 \\
\hline ctio04 & CTIO 4-m & May $16-20,2004$ & 13 \\
\hline kpno04 & KPNO 4-m & Jan. $11-12,2005$ & 3 \\
\hline ctio05 & CTIO 4-m & Jun. $7-11,2005$ & 6 \\
\hline Total & & & 65 \\
\hline
\end{tabular}

${ }^{*}$ CTIO observations in 2002 were completely clouded.

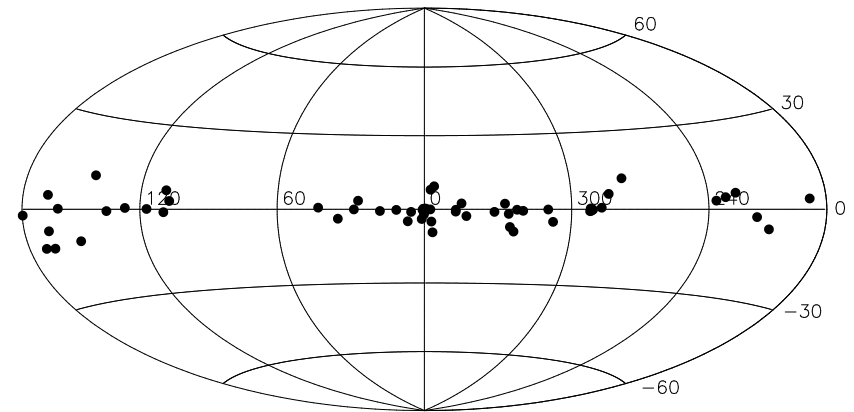

FIG. 1.- Observed ChaMPlane Mosaic fields in Galactic coordinates. Several fields in the Galactic center region are unresolved on this figure (see Grindlav et al. (2005) for an expanded view of the galactic center region fields.).

$8 \mathrm{k} \times 8 \mathrm{k}$ CCD array, which consists of eight $2048 \times 4096$ SITe CCDs. The pixel size is $15 \mu \mathrm{m}\left(0.26^{\prime \prime}\right.$ at the $4-\mathrm{m}$ telescope), which gives adequate resolution. It provides a field of view of $36^{\prime} \times 36^{\prime}$, which covers the full Chandra/ACIS FoV. Images were taken with filters of Johnson V, R, I and $\mathrm{H} \alpha(80 \AA$ FWHM centered on the $\mathrm{H} \alpha$ line).

To prepare the observation, the center of each Mosaic field (i.e. the telescope pointing) was carefully positioned so that the Mosaic covers all the active ACIS chips (typically, six ACIS chips are turned on). For the Mosaic fields covering multiple Chandra observations, their centers were positioned so that the Mosaic can cover maximum numbers of active ACIS chips possible.

\subsection{Observations}

Table 1 lists all the ChaMPlane Optical Imaging runs conducted. Table 2 is a complete list of all 65 observed ChaMPlane Mosaic fields. The name of each field is based on the main Chandra observation covered by that Mosaic field, except for fields GC1 - GC6, which are our Galactic Center mapping of 2.2 square degrees that covers 58 Chandra ACIS observations in Cycles 1-6 (including 20 SgrA* (Muno et al. 2003), 30 Galactic Center Survey (Wang et al. 2002) and 8 other observations in the Galactic Center region). Figure 1 shows these 65 fields in Galactic coordinates.

Exposure times were targeted to obtain "shallow" exposures to sample the bright sources in the field and "deep" exposures to reach the survey goal of $\sim 24$ mag for $5 \%$ photometry in the R filter and $10 \%$ photometry in the other filters. This is to ensure that our primary measurement of $\mathrm{H} \alpha-\mathrm{R}$, to search for $\mathrm{H} \alpha$ bright optical counterparts, is not compromised by limited sensitivity in the narrow-band $\mathrm{H} \alpha$ filter. This sensitivity limit is computed assuming average seeing $\left(\sim 1.0^{\prime \prime}\right)$, airmass $(\sim 1.2)$, and lunar phase $(\sim 4$ days). Shallow exposures were 2 seconds for each of the V, R, I filters and 30 seconds for the $\mathrm{H} \alpha$ filter; deep exposures were 900 seconds each for $\mathrm{V}$ and I, 1200 seconds for $\mathrm{R}$, and 7500 seconds for $\mathrm{H} \alpha$. Each of the long exposures were divided into 5 dithered sub-exposures to prevent chip gaps and bad columns in the data and to provide cosmic-ray rejection. Stars usually saturate at $\sim 12 \mathrm{mag}$ in the shallow images and $\sim 17$ mag in the deep ones. Considering overhead time at the telescope, each field takes $3.5-4$ hours, allowing us to observe three fields per night. Absolute minimal observations require the deep $\mathrm{R}$ and $\mathrm{H} \alpha$ exposures.

Standard calibration images (biases, dome flats) are taken at the telescope daily before observing. Ideal sky flats are usually constructed from object frames after eliminating all the stars. In the Galactic plane, however, the stellar density is too high for this process, so instead we obtained twilight flats. Sky flats are critically important for the I and $\mathrm{H} \alpha$ images from the KPNO 4-m to remove a pupil ghost caused by light back-scattered from the telescope optics that affects the four inner Mosaic CCDs. Dark images are not needed for this project as the dark current is very low $\left(\sim 5 e^{-} / \mathrm{pixel} / \mathrm{hr}\right.$ for Mosaic-I and $\sim<2 e^{-} / \mathrm{pixel} / \mathrm{hr}$ for Mosaic-II).

\section{MOSAIC DATA REDUCTION}

The data reduction of Mosaic images is done using the Mosaic Data Reduction package (MSCRED) ${ }^{5}$ of IRAF $^{6}$. This section summarizes our reduction process, relying heavily on the detailed reduction description of Jannuzi et al. $^{7}$ for the NOAO Deep Wide Field Survey.

\subsection{CCD reduction}

The raw data are reduced using the $I R A F /$ mscred/ccdproc package, including standard CCD corrections. For the Mosaic-II data, the data from the two amplifiers of the same CCD are merged. The Mosaic-I images are affected by the pupil ghost described in the Mosaic User Manual ${ }^{8}$ and the sky flats are used to remove the ghost at this juncture. Each reduced image is then processed to remove cosmic rays and to fix the bad pixels and columns.

\subsection{Astrometry}

Astrometry was performed on each image using IRAF $/ \mathrm{mscred} / \mathrm{msccmatch}$ which allows for zero-point shifts, scale changes, and axis rotations. It also corrects for atmospheric refraction effects. Of the two World Coordinate System (WCS) catalogs accessible by

\footnotetext{
5 http://iraf.noao.edu/iraf/web/irafnews/apr98/irafnews.21.html

${ }^{6}$ IRAF (Imaging Reduction and Analysis Facility) is distributed by the National Optical Astronomy Observatory, which is operated by the Association of Universities for Research in Astronomy, Inc., under cooperative agreement with the National Science Foundation.

${ }^{7}$ http://www.noao.edu/noao/noaodeep/ReductionOpt/frames.html

8 http://www.noao.edu/kpno/mosaic/manual/man_sep04.pdf
} 
TABLE 2

65 ChaMPlane Mosaic Fields (SORTed By RA)

\begin{tabular}{|c|c|c|c|c|c|c|c|}
\hline$\overline{N^{a}}$ & $\overline{\text { Code }}$ & Field & RA(J2000) & 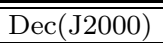 & $\overline{l l\left({ }^{\circ}\right)}$ & $\overline{b\left(\left(^{\circ}\right)\right.}$ & $\overline{\overline{\mathrm{N}_{\mathrm{H}} / 10^{22 \mathrm{~b}}}}$ \\
\hline 26 & kpno02 & G127.1+0.5 & $01: 28: 00.00$ & $+63: 03: 11.7$ & 127.06023 & 0.47507 & 0.869 \\
\hline 58 & kpno04 & MAFFEI1 & $02: 36: 49.80$ & $+59: 36: 19.0$ & 135.90926 & -0.58435 & 0.591 \\
\hline 11 & kpno00 & GKPERSEI & $03: 30: 37.89$ & $+43: 51: 15.8$ & 150.90053 & -10.20409 & 0.181 \\
\hline 12 & kpno00 & GROJ0422+32 & $04: 21: 20.40$ & $+32: 55: 37.9$ & 165.81091 & -11.95536 & 0.189 \\
\hline 13 & kpno00 & NGC1569 & $04: 30: 25.00$ & $+64: 44: 53.0$ & 143.72842 & 11.14309 & 0.412 \\
\hline 23 & kpno01 & $3 \mathrm{C} 123$ & $04: 36: 28.00$ & $+29: 37: 49.0$ & 170.52481 & -11.78690 & 0.659 \\
\hline 42 & kpno03 & AFGL618 & $04: 42: 23.39$ & $+36: 04: 35.3$ & 166.40825 & -6.62965 & 0.462 \\
\hline 24 & kpno01 & 3C129 & $04: 49: 19.20$ & $+45: 02: 30.0$ & 160.42311 & 0.18431 & 0.644 \\
\hline 27 & kpno02 & G166.0+4.2 & $05: 27: 12.06$ & $+42: 54: 05.1$ & 166.21402 & 4.36698 & 0.378 \\
\hline 28 & kpno02 & PSRJ0538+2817 & $05: 38: 00.54$ & $+28: 14: 24.0$ & 179.70937 & -1.78641 & 0.759 \\
\hline 43 & kpno03 & 1SAXJ0618.0+2227 & $06: 18: 18.70$ & $+22: 29: 20.8$ & 189.24487 & 3.20883 & 1.045 \\
\hline 1 & ctio00 & A0620-00 & $06: 23: 15.70$ & $-00: 18: 20.7$ & 209.98050 & -6.40597 & 0.292 \\
\hline 29 & kpno02 & MADDALENA'SCLOUD & $06: 49: 24.78$ & $-04: 34: 07.2$ & 216.77023 & -2.53092 & 0.944 \\
\hline 30 & kpno02 & M1-16 & $07: 37: 14.89$ & $-09: 41: 07.6$ & 226.82573 & 5.59410 & 0.130 \\
\hline 59 & kpno04 & $\mathrm{OH} 231.8+4.2$ & $07: 42: 01.49$ & $-14: 45: 18.0$ & 231.84038 & 4.14583 & 0.428 \\
\hline 2 & ctio00 & PKS0745-191 & $07: 47: 16.70$ & $-19: 15: 05.0$ & 236.37517 & 3.00117 & 0.306 \\
\hline 3 & ctio00 & NGC3256 & $10: 28: 41.70$ & $-43: 59: 18.3$ & 277.54922 & 11.73626 & 0.068 \\
\hline 14 & ctio01 & V382VELORUM1999 & $10: 44: 48.00$ & $-52: 18: 18.0$ & 284.10977 & 5.87716 & 0.258 \\
\hline 60 & ctio05 & PSRB1046-58 & 10:48:00.00 & $-58: 28: 48.0$ & 287.37619 & 0.61334 & 0.684 \\
\hline 31 & ctio03 & MSH11-62 & $11: 11: 57.38$ & $-60: 41: 28.0$ & 291.05115 & -0.13479 & 0.637 \\
\hline 15 & ctio01 & NGC3603 & $11: 15: 49.80$ & $-61: 17: 23.0$ & 291.70792 & -0.51893 & 16.683 \\
\hline 32 & ctio03 & G292.2-0.5 & $11: 19: 45.94$ & $-61: 40: 13.5$ & 292.28198 & -0.70890 & 2.162 \\
\hline 33 & ctio03 & CENX-3 & $11: 20: 49.67$ & $-60: 37: 04.3$ & 292.03949 & 0.32333 & 0.665 \\
\hline 61 & ctio05 & MYCN18 & $13: 39: 57.60$ & $-67: 20: 24.0$ & 307.59271 & -4.90835 & 0.317 \\
\hline 44 & ctio04 & G309.8+0.0 & $13: 50: 12.00$ & $-62: 09: 36.0$ & 309.73349 & -0.06672 & 7.336 \\
\hline 45 & ctio04 & PSRJ1509-5850 & 15:09:36.00 & $-58: 48: 18.0$ & 320.01083 & -0.59235 & 6.358 \\
\hline 46 & ctio04 & G322.5-0.1 & $15: 24: 00.00$ & $-57: 06: 53.2$ & 322.52438 & -0.16385 & 4.416 \\
\hline 16 & ctio01 & $4 \mathrm{U} 1538-52$ & $15: 42: 01.40$ & $-52: 18: 22.0$ & 327.42334 & 2.26117 & 1.319 \\
\hline 47 & ctio04 & XTEJ1550-564 & 15:51:01.07 & $-56: 27: 16.6$ & 325.90033 & -1.81329 & 0.944 \\
\hline 62 & ctio05 & ABELL3627 & $16: 13: 45.60$ & $-60: 48: 36.0$ & 325.24203 & -7.03382 & 0.121 \\
\hline 34 & ctio03 & 1RXSJ161411.3-630657 & $16: 14: 53.00$ & $-63: 12: 57.0$ & 323.64777 & -8.85384 & 0.075 \\
\hline 48 & ctio04 & MZ3 & $16: 17: 09.70$ & $-51: 59: 30.2$ & 331.71680 & -1.00867 & 1.132 \\
\hline 4 & ctio00 & GROJ1655-40 & $16: 54: 28.90$ & $-39: 51: 31.7$ & 345.02972 & 2.37637 & 0.638 \\
\hline 49 & ctio04 & MARS & $17: 00: 48.53$ & $-26: 58: 24.2$ & 356.04932 & 9.28549 & 0.132 \\
\hline 50 & ctio04 & XTEJ1709-267 & $17: 09: 36.71$ & $-26: 36: 53.0$ & 357.51978 & 7.91659 & 0.341 \\
\hline 17 & ctio01 & PSRB1706-44 & $17: 09: 42.00$ & $-44: 30: 45.0$ & 343.07489 & -2.69993 & 1.319 \\
\hline 5 & ctio00 & G347.5-0.5a & $17: 12: 16.10$ & $-39: 34: 39.6$ & 347.33688 & -0.16436 & 4.895 \\
\hline 6 & ctio00 & G347.5-0.5b & $17: 15: 36.00$ & $-39: 58: 36.0$ & 347.38821 & -0.91690 & 1.794 \\
\hline 51 & ctio04 & TORNADO & $17: 40: 00.00$ & $-30: 57: 59.2$ & 357.63300 & -0.03307 & 11.271 \\
\hline 35 & ctio03 & GC5 & $17: 43: 04.80$ & $-29: 37: 48.0$ & 359.11887 & 0.10892 & 15.853 \\
\hline 36 & ctio03 & $\mathrm{GC} 2$ & $17: 43: 33.12$ & $-29: 01: 48.0$ & 359.68350 & 0.33651 & 4.930 \\
\hline 37 & ctio03 & GC3 & $17: 45: 44.88$ & $-28: 25: 48.0$ & 0.44666 & 0.23978 & 17.838 \\
\hline 38 & ctio03 & GC6 & $17: 45: 49.20$ & $-29: 37: 48.0$ & 359.43039 & -0.39852 & 10.212 \\
\hline 7 & ctio00 & SGRA* & $17: 46: 11.20$ & $-28: 53: 52.0$ & 0.09729 & -0.08582 & 54.518 \\
\hline 39 & ctio03 & GC1 & $17: 46: 16.32$ & $-29: 01: 48.0$ & 359.99405 & -0.17051 & 36.045 \\
\hline 8 & ctio00 & SGRB2 & $17: 46: 43.10$ & $-28: 29: 42.1$ & 0.50199 & 0.02380 & 65.053 \\
\hline 9 & ctio00 & GALACTICCENTERARC & $17: 47: 08.60$ & $-28: 53: 36.0$ & 0.20979 & -0.26248 & 20.284 \\
\hline 40 & ctio03 & GC4 & $17: 48: 27.60$ & $-28: 25: 48.0$ & 0.75573 & -0.27009 & 30.783 \\
\hline 63 & ctio05 & LIMITINGWINDOW & $17: 51: 48.00$ & $-29: 34: 12.0$ & 0.15160 & -1.48171 & 0.704 \\
\hline 52 & ctio04 & STANEKWINDOW & $17: 54: 24.42$ & $-29: 49: 16.3$ & 0.22242 & -2.09703 & 0.478 \\
\hline 53 & ctio04 & $4 \mathrm{U} 1755-33$ & $17: 58: 49.20$ & $-33: 51: 00.0$ & 357.19409 & -4.92093 & 0.399 \\
\hline 18 & ctio01 & PSRB1757-24 & $18: 01: 48.00$ & $-24: 49: 39.0$ & 5.37027 & -1.02458 & 4.030 \\
\hline 54 & ctio04 & BAADE'SWINDOW & $18: 03: 36.00$ & $-29: 57: 50.7$ & 1.08909 & -3.89739 & 0.321 \\
\hline 55 & ctio04 & G11.4-0.1 & $18: 11: 20.40$ & $-19: 14: 24.0$ & 11.32621 & -0.22863 & 8.865 \\
\hline 19 & ctio01 & PSR1813-36 & $18: 16: 25.00$ & $-36: 20: 29.5$ & 356.70163 & -9.27033 & 0.092 \\
\hline 64 & ctio05 & V4641SGR & $18: 19: 21.38$ & $-25: 27: 00.0$ & 6.73546 & -4.80812 & 0.289 \\
\hline 56 & ctio04 & PSRB1823-13 & $18: 26: 00.00$ & $-13: 37: 12.0$ & 17.94011 & -0.66268 & 7.538 \\
\hline 20 & ctio01 & MWC297 & $18: 27: 59.80$ & $-03: 51: 02.6$ & 26.82325 & 3.44240 & 6.963 \\
\hline 41 & ctio03 & GALACTICPLANE & $18: 43: 27.72$ & $-03: 58: 12.0$ & 28.49013 & -0.03869 & 17.281 \\
\hline 21 & ctio01 & SGR1900+14 & $19: 07: 38.30$ & $+09: 18: 08.1$ & 43.04858 & 0.66903 & 2.784 \\
\hline 65 & ctio05 & $1 \mathrm{H} 1905+000$ & $19: 08: 36.00$ & $+00: 11: 24.0$ & 35.06054 & -3.73080 & 0.359 \\
\hline 10 & kpno00 & $\mathrm{B} 2224+65$ & $22: 25: 13.22$ & $+65: 32: 45.2$ & 108.55382 & 6.84190 & 0.460 \\
\hline 22 & kpno01 & $3 \mathrm{EGJ} 2227+6122$ & $22: 29: 17.00$ & $+61: 19: 00.9$ & 106.70962 & 3.00593 & 1.012 \\
\hline 57 & kpno04 & CTB-109LOBE & $23: 02: 18.85$ & $+58: 54: 40.1$ & 109.23905 & -1.02858 & 1.032 \\
\hline 25 & kpno02 & G116.9+0.2 & $23: 59: 12.53$ & $+62: 24: 00.0$ & 116.92374 & 0.13922 & 0.461 \\
\hline
\end{tabular}

${ }^{\mathrm{a}} \mathrm{N}$ is the ID of the Mosaic field, which is in chronological order of the observations.

${ }^{\mathrm{b}}$ The full column $\mathrm{N}_{\mathrm{H}} / 10^{22}$, based on Schlegel et al. (1998). Thus the $\mathrm{N}_{\mathrm{H}}$ values are overestimated for most Galactic plane fields since the Schlegel values for $\mathrm{N}_{\mathrm{H}}$ are for the full Galactic absorption whereas optical counterparts for most sources detected are in the foreground. 
msccmatch - the US Naval Observatory A2.0 catalog (NOAO:USNO-A2) ${ }^{9}$ and the Hubble Space Telescope Guide Star Catalog Version 2 (GSC2@STSCI) ${ }^{10}$ - we chose to use the USNO-A2 catalog because it provides complete coverage of the sky. Following coordinate registration, we then re-project each image to a defined tangent plane using mscimage, allowing us to stack multiple images. All re-projections are carried out using sinc function interpolation because it preserves all spatial frequencies and is the mathematically correct interpolation method for well-sampled data. After the projection, each image needs to have residual large-scale (field wide) gradients in the sky background removed. This is done by using mscskysub on each projected image.

\subsection{Image Stacking}

Before stacking the images, the relative intensity scales are adjusted using mscimatch on each group of images to be stacked. Finally the images are stacked using $m s c$ stack with median-combine, which further removes the cosmic rays, bad pixels and other defects. Typically we end up with eight final images for each field: shallow, single images of the $\mathrm{V}, \mathrm{R}, \mathrm{I}$ and $\mathrm{H} \alpha$ filters and corresponding stacked deep images. These images are available from the ChaMPlane website (see Grindlav et al. (2005)).

\section{PHOTOMETRY AND PHOTOMETRIC CALIBRATION}

Photometric analysis uses the standard approach for DAOphot in the IRAF package noao.digiphot.daophot ${ }^{11}$. The source lists are generated from all eight images using the task daofind, with different threshold, i.e. the detected counts in units of sky RMS. For shallow images, the threshold is set high $(\sim 25)$ to detect sources brighter than 19 mag; for the deep images, the threshold is set low $(\sim 4)$ to detect all possible sources. A comprehensive Master Star List for each field is generated by merging these eight source lists and removing multiple detections, defined as sources with positions within 1 pixel $\left(0.26^{\prime \prime}\right)$ of each other. So the source positions in each master star list are on the integer pixel grid. Additional duplications will be removed while re-centering during the PSF fit photometry. The final star positions are determined by the center of their PSF. For each image, 1000 PSF star candidates were carefully selected, based on their sharpness, sround and ground values from daofind. These candidates should uniformly cover the entire field and are bright enough but not saturated. This candidate list is fed into the task psfselect, which selects the PSF stars. A Point Spread Function is calculated for each image from these PSF stars, using psf, that is constant over the whole field. Aperture photometry and PSF fit photometry (DAOphot) are carried out using tasks phot and allstar, respectively, on the Master Star List.

The photometric calibration was obtained from CCD images of ChaMPlane fields and Landolt standards (Landolt 1992) on photometric nights using the FLWO

\footnotetext{
${ }^{9}$ http://tdc-www.harvard.edu/software/catalogs/ua2.html

$10 \mathrm{http}: / /$ www-gsss.stsci.edu/gsc/gsc2/GSC2home.htm

11 http://iraf.noao.edu/docs/photom.html: "A User's Guide to Stellar CCD Photometry with IRAF", P. Massey and L. Davis, 1992; "A Reference Guide to the IRAF/DAOPHOT Package", L. Davis, 1994.
}

1.2-m (north) and CTIO 1.3-m (south) telescopes, so we could spend all our 4-m time on the ChaMPlane fields. Typically we take the V, R, I images of those ChaMPlane fields to be calibrated and 2-3 Landolt fields (with 5 to a couple dozen Landolt standard stars per field) at different airmass. Standard calibration procedures were used (IRAF/noao.digiphot.photcal) to compute the photometric transformations and to determine the V, R, I magnitude standard. For the $\mathrm{H} \alpha$ magnitudes, we define the median point of $\mathrm{H} \alpha-\mathrm{R} \equiv 0$.

\section{OPTICAL CATALOG}

Even with DAOphot, many false detections (e.g. near the edges, gaps and saturated stars, under the shadow of bright stars, etc.) could still survive the PSF fitting process. To select the real sources, we choose objects with the PSF fitting parameter: sharpness $>-1$. Results with sharpness $\leqslant-1$ are usually too sharp to be real sources but false detections. The above selection includes both point and extended sources. Point sources usually have $\mid$ sharpness $\mid<1$; while extended sources (e.g. galaxies) have sharpness $\geqslant 1$. After selection, for each field, a catalog is established based on the DAOphot results; each entry in the catalog includes the source ID, RA and Dec, X and Y position on the image, V, R, I and $\mathrm{H} \alpha$ magnitudes and their errors, and the PSF fitting parameters $\chi^{2}$ and sharpness.

All the Mosaic optical catalogs will be in the ChaMPlane Online Database along with the Chandra source optical counterparts, available from the ChaMPlane website 3 and NOAO Science Archive ${ }^{12}$.

\section{ASTROMETRIC ACCURACY}

We examined the astrometric accuracy of our optical catalog using overlapping areas of the Mosaic fields in our Galactic Center (GC) mapping. We matched the positions of identical stars appearing in different Mosaic fields. Figure 2 shows the results from a rectangular $\left(4^{\prime}\right.$ in RA and $36^{\prime}$ in Dec) overlapping area between the GC1 and GC2 fields (see Table 2). The upper left panel shows the offsets of the identical stars between the two fields; the upper right panel is a histogram displaying the $\mathrm{R}$ magnitude difference of those stars. The two lower panels display the histograms of the RA and Dec offsets of these stars. The offsets and their errors are a little larger in RA than in Dec, because the overlapping area in RA is small (matching one side of one field to the other side of another field) while the overlapping area in Dec covers the entire Mosaic length. The standard deviation of the position mismatch is $0.0984^{\prime \prime}$ in RA and $0.0826^{\prime \prime}$ in Dec and represents the random precision of individual stars. Note that this is the worse-case scenario because we are comparing the astrometry near the chip edge and at opposite side of the Mosaic camera. The astrometry improves toward the center of the detector. Even so, the standard deviation is still less than $0.1^{\prime \prime}$, which is contributed by two Mosaic fields. Assuming each one of them has the same contribution to the mismatch, the precision of each field should be $0.07^{\prime \prime}$. To be conservative, we assign each individual star with an astrometric accuracy of $0.1^{\prime \prime}(1-\sigma)$. This value is used in Section 8 to identify optical counterparts of Chandra sources.

\footnotetext{
12 http://archive.noao.edu/nsa/
} 

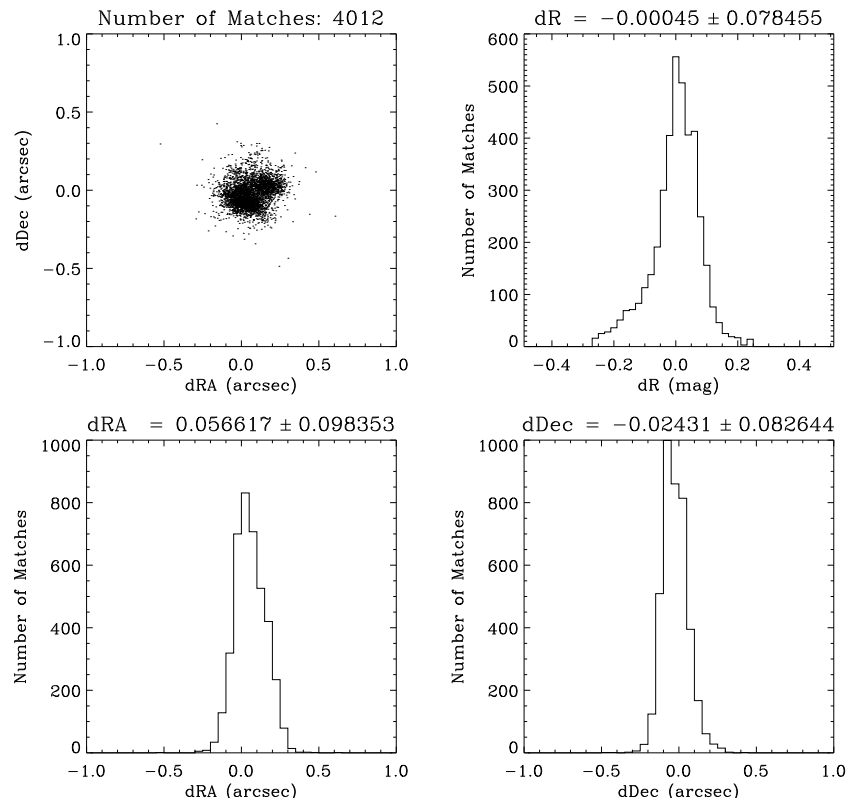

FIG. 2.- The astrometric precision of the Mosaic photometry. The upper left panel shows the offset of the same stars between the GC1 and GC2 fields in their overlapping area. The upper right panel is a histogram showing the $\mathrm{R}$ magnitude difference of the same stars between the GC1 and GC2 optical catalogs. The two lower panels are the histograms of the offset in RA and Dec of these stars.

\section{7. $\mathrm{H} \alpha$ EMISSION OBJECTS}

Accretion-powered binaries are characterized by their (often double peaked) hydrogen Balmer series emission lines generated in the outer region of their accretion disks. Among them, the $\mathrm{H} \alpha$ line is the most prominent. Therefore we have designed our Mosaic observations (see 2.21) to spend $\sim 6 \times$ longer exposures in $\mathrm{H} \alpha$ than in $\mathrm{R}$ to allow maximum sensitivity to $\mathrm{H} \alpha-\mathrm{R}$ colors (see below). Since the Mosaic CCDs cover about 5 times more sky than ACIS-I, we expect and found on average $80 \%$ of the $\mathrm{H} \alpha$ emission objects lying outside the ACIS FoV.

To select sources with significant $\mathrm{H} \alpha$ excess, we first define the signal-to-noise ratio, $S / N$, in terms of the flux ratio, $F$, between $\mathrm{H} \alpha$ and $\mathrm{R}$ bands as follows.

$$
\begin{aligned}
S / N & =\frac{F-F_{0}}{\Delta F} \\
F & = \begin{cases}\frac{f_{H \alpha}}{f_{R}} & \text { if } \mathrm{H} \alpha<\mathrm{R}(\mathrm{H} \alpha \text { emission }) \\
F_{0} & \text { if } \mathrm{H} \alpha=\mathrm{R} \\
\frac{f_{R}}{f_{H \alpha}} F_{0}^{2} & \text { if } \mathrm{H} \alpha>\mathrm{R}(\mathrm{H} \alpha \text { absorption })\end{cases}
\end{aligned}
$$

where $\mathrm{H} \alpha$ and $\mathrm{R}$ are the magnitudes in the $\mathrm{H} \alpha$ and $\mathrm{R}$ bands; $f_{H \alpha}$ and $f_{R}$ are their fluxes; and $F_{0}$ corresponds to the median flux ratio of $\frac{f_{H \alpha_{0}}}{f_{R_{0}}}$ in the stellar sample, i.e. when $\mathrm{H} \alpha=$ R. ${ }^{13}$ Since by definition $F$ is always greater than or equal to $F_{0}, S / N$ is always greater than or equal to zero. The uncertainty, i.e. noise, of $F$ is $\Delta F$, which by definition is also always positive.

Since

$$
|\mathrm{H} \alpha-\mathrm{R}|=2.5 \log \frac{F}{F_{0}}
$$

13 A factor of $F_{0}^{2}$ on the right hand side of Eq. 2) is needed to preserve the continuity of the function $F$.

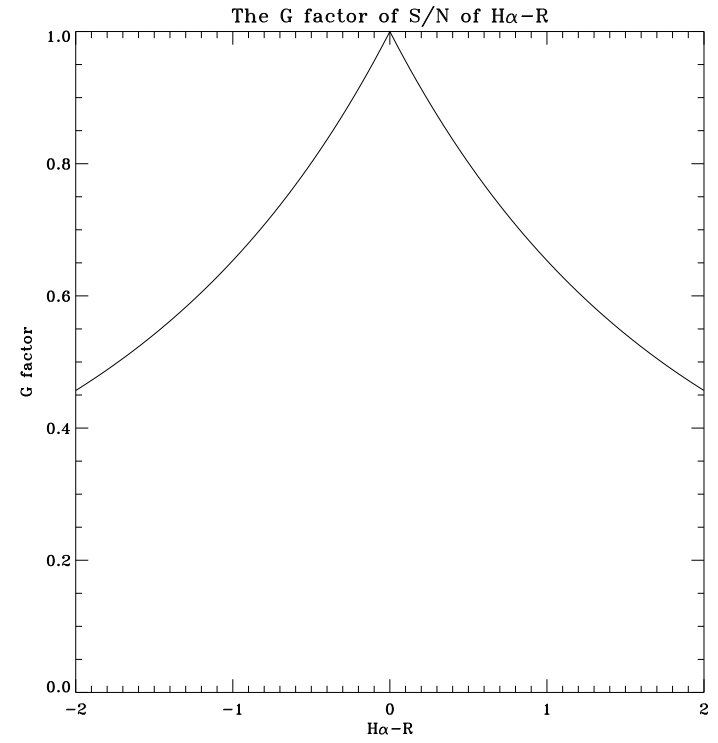

FIG. 3.- The G factor of signal-to-noise ratio, $S / N$, in terms of the flux ratio, $F$, between $\mathrm{H} \alpha$ and $\mathrm{R}$ bands.

$$
\Delta|\mathrm{H} \alpha-\mathrm{R}|=\frac{2.5}{\ln 10} \frac{\Delta F}{F}
$$

where $\Delta|\mathrm{H} \alpha-\mathrm{R}|=\sqrt{(\Delta H \alpha)^{2}+(\Delta R)^{2}}$.

Therefore

$$
\begin{aligned}
S / N & =\left(1-10^{-0.4|\mathrm{H} \alpha-\mathrm{R}|}\right) \frac{F}{\Delta F} \\
& =G \cdot \frac{|\mathrm{H} \alpha-\mathrm{R}|}{\Delta|\mathrm{H} \alpha-\mathrm{R}|}
\end{aligned}
$$

where $G=\frac{2.5}{\ln 10} \cdot \frac{1-10^{-0.4|\mathrm{H} \alpha-\mathrm{R}|}}{|\mathrm{H} \alpha-\mathrm{R}|}$ is the so-called $G$ factor, which is a function of $|\mathrm{H} \alpha-\mathrm{R}|$ as shown in Figure 3

Having defined the $S / N$, we select possible $\mathrm{H} \alpha$ emission objects using criteria:

$$
\begin{gathered}
H \alpha-R \leqslant-0.3 \\
S / N \geqslant 5
\end{gathered}
$$

The first criterion (Eq. (7)) selects CVs instead of dMe stars. Figure 4 shows the composite transmission curves of the Mosaic $\mathrm{R}$ and $\mathrm{H} \alpha$ filters plus the CCD quantum efficiency, and $\mathrm{H} \alpha-\mathrm{R}$ as a function of the $\mathrm{H} \alpha$ emission equivalent width (EW), assuming a flat continuum. By choosing $\mathrm{H} \alpha-\mathrm{R} \leqslant-0.3$ we are selecting objects with $\mathrm{H} \alpha \mathrm{EW}$ greater than $28 \AA$. But the relation between $\mathrm{H} \alpha-\mathrm{R}$ and $\mathrm{H} \alpha \mathrm{EW}$ also depends on the spectral type. Figure 5 shows the $\mathrm{H} \alpha-\mathrm{R}$ as a function of the $\mathrm{H} \alpha \mathrm{EW}$ for different main sequence stars. ${ }^{14}$ Most dMe stars have $\mathrm{EW}(\mathrm{H} \alpha)<10 \AA$ (Mochnacki et al. 2002), whereas most CVs (except dwarf novae in outburst) have $\mathrm{EW}(\mathrm{H} \alpha)>10 \AA$ (William 1983; Warner 1995; Szkodv et al. 2002, 2003, 2004, 2005). The criterion of $\mathrm{H} \alpha-\mathrm{R} \leqslant-0.3$ further distances the selection from the large numbers of dMe stars in each field. The second criterion (Eq. (8)) discriminates noise in the faint end. Because the G-factor is always less than $1, S / N$ is always less than $\frac{|\mathrm{H} \alpha-\mathrm{R}|}{\Delta|\mathrm{H} \alpha-\mathrm{R}|}$. Thus using $S / N$ instead of $\frac{|\mathrm{H} \alpha-\mathrm{R}|}{\Delta|\mathrm{H} \alpha-\mathrm{R}|}$ makes the selection more restrictive. Finally, we visually

14 Based on Kurucz Models (Dr. R. Kurucz, CD-ROM No. 13, GSFC) from http://garnet.stsci.edu/STIS/stis_models.html 

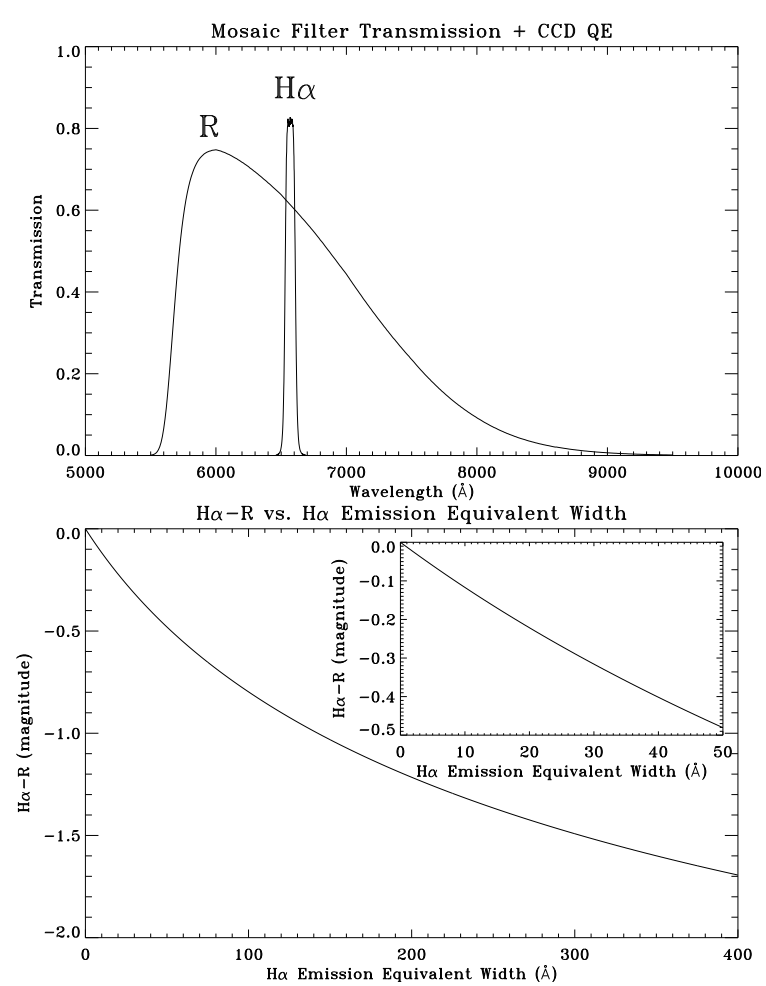

FIG. 4. - Top panel: the composite transmission curves of the Mosaic $\mathrm{R}$ and $\mathrm{H} \alpha$ filters plus the CCD quantum efficiency. Bottom panel: $\mathrm{H} \alpha-\mathrm{R}$ as a function of the $\mathrm{H} \alpha$ emission equivalent width (assuming a flat continuum).

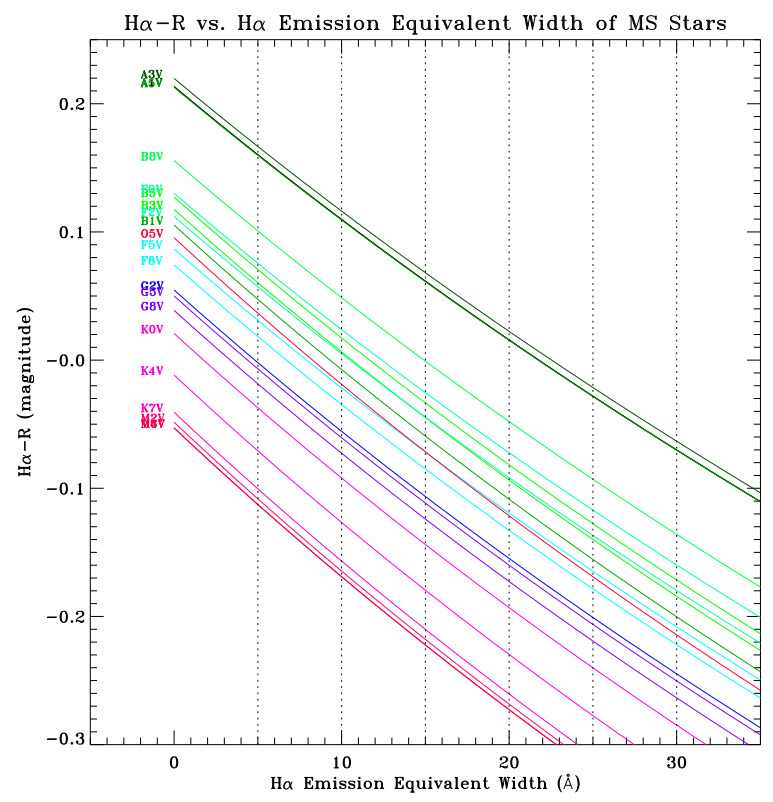

Fig. 5.- $\mathrm{H} \alpha-\mathrm{R}$ as a function of the $\mathrm{H} \alpha$ emission equivalent width of main sequence stars for the Mosaic $\mathrm{H} \alpha$ and $\mathrm{R}$ filters. (See electronic ApJ for the colored version.)

examine all the $\mathrm{H} \alpha$ objects on the $\mathrm{H} \alpha$ and $\mathrm{R}$ image pairs to eliminate false detections.

\section{CHANDRA OPTICAL COUNTERPARTS}

\subsection{X-ray source position error}

Serendipitous X-ray sources in the ChaMPlane fields are detected from the Chandra archival data, using the methods (the ChaMPlane X-ray processing routines
XPIPE and PXP) described in Hong et al. (2005). Each Chandra source has a position error that depends on the net source counts and off-axis angle from the aimpoint. The $95 \%$ confidence error radii, $r_{x(95 \%)}$, are calculated as a function of net counts and off-axis angle, according to an empirical formula based on the results of raytrace simulations and XPIPE detections (Hong et al. 2005).

\subsection{X-ray source boresight correction}

Other than individual random errors, the Chandra sources also have a small systematic position offset, usually less than $1^{\prime \prime}$ relative to the optical positions. Before matching X-ray and optical sources, the X-ray positions are corrected for this boresight difference $(\Delta \alpha, \Delta \delta)$ that is determined for each observation separately.

To compute the boresight correction we first select Chandra sources with well-determined positions, i.e. $r_{x(95 \%)}$ smaller than typically $1^{\prime \prime}$ to $2^{\prime \prime}$. If in the end it turns out that the final boresight is based on only a few (2 or 3) pairs of X-ray/optical matches, the limit on $r_{x(95 \%)}$ is increased. Optical sources are selected from the Optical Catalog to have $R<23$ to guarantee good positions. If no $R$ magnitude is available, we require that the source is brighter than 23 in either $V, I$ or $H \alpha$ - in that order of priority.

The resulting X-ray and optical source lists are crosscorrelated. Initially we accept matches inside a large match radius which combines statistical and systematic errors for a combined $2 \sigma$ value:

$$
R_{0}=2 \times \sqrt{r_{x}^{2}+r_{o p t}^{2}+r_{x s y s}^{2}}
$$

where

$r_{x}=r_{x(95 \%)} / 1.95996$, is the $1-\sigma$ random error on X-ray positions, assuming the errors follow a Gaussian distribution;

$r_{\text {opt }}=0.1^{\prime \prime}$ is the random error of individual optical positions with respect to the astrometric frame (see section 6); and

$r_{x y s}=0.6^{\prime \prime} / 1.6449$, is the $1-\sigma$ typical boresight offset of X-ray positions $\left(0.6^{\prime \prime}\right.$ is the $90 \%$ absolute accuracy on Chandra positions $\left.{ }^{15}\right)$.

A weighted (by $\left.\left.1 / r_{x(95 \%)}\right)^{2}\right)$ average offset in RA $(\Delta \alpha)$ and Dec $(\Delta \delta)$ (defined as the X-ray position minus the optical position) is computed after excluding X-ray sources with more than one possible (i.e. within $R_{0}$ ) optical counterpart. Errors on the offsets, $\Delta \alpha_{e r r}$ and $\Delta \delta_{\text {err }}$, are computed as the square-root of the weighted variance in $\Delta \alpha$ and $\Delta \delta$ divided by the number of matches. $\mathrm{X}$-ray/optical matches whose individual offsets lie more than $3 \times \Delta \alpha_{e r r}$ or $3 \times \Delta \delta_{\text {err }}$ from the average are rejected, and the weighted average is re-computed.

After correcting the X-ray positions for $\Delta \alpha$ and $\Delta \delta$, the cross-correlation is repeated. In the second and consecutive iterations the match-radius is set as follows:

$$
R_{1}=2 \times \sqrt{r_{x}^{2}+r_{\text {opt }}^{2}+r_{\text {bore }}^{2}}
$$

where $r_{b o r e}$ is the greater of $\Delta \alpha_{e r r}$ or $\Delta \delta_{e r r}$ in the previous iteration.

The above steps are iterated until the boresight correction converges to a stable solution, which is reached in

15 http://cxc.harvard.edu/cal/docs/cal_present_status.html, http://cxc.harvard.edu/cal/ASPECT/celmon/ 
typically 2 to 4 passes. The result is inspected visually against a Mosaic image; optical sources with positions that look unreliable (e.g. the position could be off from the center-of-light, or there could be a nearby undetected source) are removed from the catalog and the calculation is redone. The final stable solution of the boresight correction is applied to all the X-ray positions. As an example, the boresight solution for the GRO J0422+32 field is $\Delta \alpha=0.21^{\prime \prime} \pm 0.059^{\prime \prime}$ and $\Delta \delta=-0.36^{\prime \prime} \pm 0.044^{\prime \prime}$.

\subsection{Optical Counterparts}

The Optical Counterparts are found around the boresight corrected X-ray positions within an error radius of:

$$
R_{\text {err }}=c \times \sqrt{r_{x}^{2}+r_{o p t}^{2}+r_{\text {bore }}^{2}}
$$

where $r_{x}$ and $r_{o p t}$ are defined in Equation $9 r_{\text {bore }}$ is the greater of $\Delta \alpha_{e r r}$ or $\Delta \delta_{e r r}$ in the final stable boresight solution; the square root expression is the combined effective matching error, $\sigma$; $c$ is a confidence-level scale factor (number of $\sigma$ ), and typically we choose $c=3$ to search for optical counterparts. For each Chandra source, we search the Mosaic optical catalog (see Section (5) within $R_{\text {err }}$ radius for its optical counterparts. If there are more than one matches within the $R_{e r r}$ radius, they are prioritized (for follow-up spectroscopy) by the matching distance and their $\mathrm{H} \alpha-\mathrm{R}$ color. This process produces the Chandra optical counterparts from the Mosaic optical catalogs, which contain all the objects with magnitude ranging from $\sim 12$ to $\sim 25$. Thus it misses out stars brighter than $\sim 12$ magnitude, which are saturated even in the short Mosaic images. To recover those possible missing bright star counterparts, we match all the Chandra sources, using the $R_{\text {err }}$ radii, with the USNOA2 and GSC2 catalogs (see Section 3.2). This process produces all the Chandra optical counterparts with magnitude brighter than $\sim 19$. When combining the results from these two matches, and removing the duplications (i.e. objects appear in both matches), we obtain a complete set of Chandra optical counterparts for a given field.

\section{RESULTS}

\subsection{ChaMPlane Optical Survey Products}

The end products of the ChaMPlane optical photometry for each Mosaic field are:

1. An optical catalog of all the objects (regardless of object type e.g., stars, CV candidates, QSOs or galaxies, as determined by followup spectroscopy) detected in the entire Mosaic field. Each entry includes the source ID, RA and Dec, $\mathrm{X}$ and Y position on the image, $\mathrm{V}, \mathrm{R}, \mathrm{I}$ and $\mathrm{H} \alpha$ magnitudes and their errors, as well as the PSF fitting parameters $\chi^{2}$ and sharpness, colors $\mathrm{V}-\mathrm{R}, \mathrm{R}-\mathrm{I}, \mathrm{H} \alpha-\mathrm{R}$ and their errors, and $\mathrm{S} / \mathrm{N}$ of $\mathrm{H} \alpha-\mathrm{R}$ (as defined in Section (7).

2. A list of $\mathrm{H} \alpha$ emission sources (as defined in Section (7) found in the entire Mosaic field.

3. A list or lists of Chandra optical counterparts (as defined in Section 8) found for every Chandra observation covered by this Mosaic field.
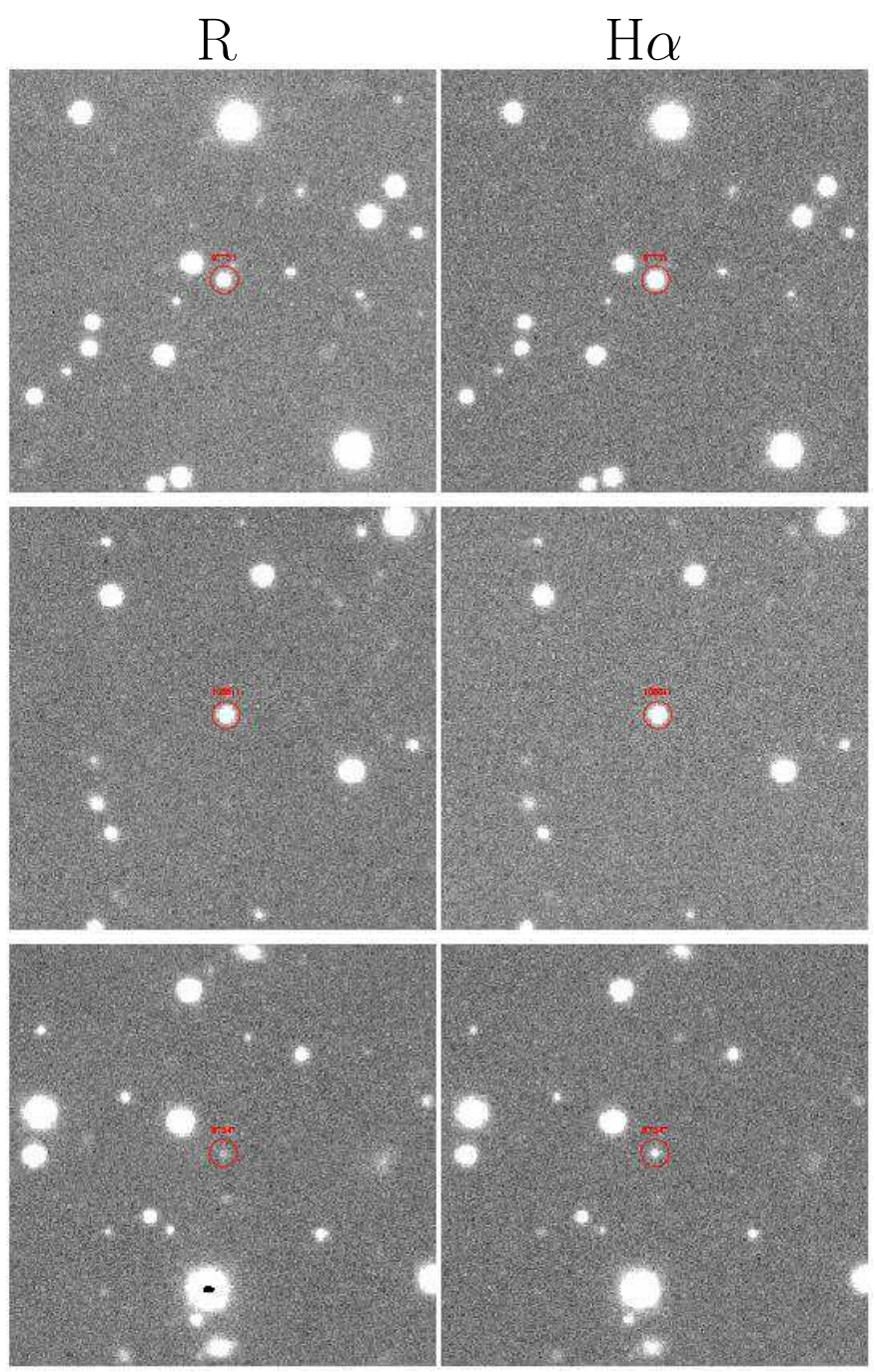

FIG. 6.- The R (left) and $\mathrm{H} \alpha$ (right) images of three $\mathrm{H} \alpha$ emission sources found in the J0422+32 Mosaic field: 1) top panels show the GRO J0422+32 itself (circled), which is obviously brighter in $\mathrm{H} \alpha$ $(\mathrm{ID}=97731, \mathrm{R}=20.80, \mathrm{H} \alpha-\mathrm{R}=-1.45) ; 2$ ) middle panels show the first CV discovered under ChaMPlane project, which is outside of the ACIS FoV (ID=108811, $\mathrm{R}=20.28, \mathrm{H} \alpha-\mathrm{R}=-0.76) ; 3$ ) bottom panels show a very bright $\mathrm{H} \alpha$ emission object $(\mathrm{ID}=87347$, $\mathrm{R}=21.67, \mathrm{H} \alpha-\mathrm{R}=-1.54)$. It is in the ACIS-I FoV. Although no X-ray emission was detected from this object, the upper limit for $F_{x} / F_{r}$ allows it to be a CV. $\left(1^{\prime} \times 1^{\prime}\right.$ FoV. $)$

\subsection{Example Results for GRO J0422+32 Field}

In this section, we use the black hole X-ray nova GRO J0422+32 Chandra field (ObsID 676, 20 ks ACIS-I observation) as an example to demonstrate the typical output of the photometry survey. The optical catalog from this Mosaic field has 29714 entries, with magnitudes ranging from 12.4 to 25.5. The catalog is accessible at the ChaMPlane website ${ }^{3}$.

Table 3 is a list of $\mathrm{H} \alpha$ emission sources with $\mathrm{H} \alpha-\mathrm{R} \leqslant-0.3$ and $\mathrm{S} / \mathrm{N} \geqslant 5$ (these criteria can, of course, be changed to select different $\mathrm{H} \alpha$ sources). There are $18 \mathrm{H} \alpha$ sources that satisfy these criteria. Among them, ID 97731 is J0422+32 itself. It has very strong $\mathrm{H} \alpha$ emission $(\mathrm{H} \alpha-\mathrm{R}=-1.45$ and $\mathrm{S} / \mathrm{N}=61.4)$ and therefore it was easily detected in our survey. ID 108811 is the first CV discovered under the ChaMPlane project. It has strong $\mathrm{H} \alpha$ emission $(\mathrm{H} \alpha-\mathrm{R}=-0.76$ and $\mathrm{S} / \mathrm{N}=41.8)$. 
TABLE 3

GRO J0422+32 FIELD: H $\alpha$ EMISSION SOURCES within the Full Mosaic Field

\begin{tabular}{|c|c|c|c|c|c|c|c|c|}
\hline OptID & $\mathrm{RA}(\mathrm{J} 2000)$ & Dec(J2000) & $\mathrm{V}(\mathrm{err})$ & $\mathrm{R}($ err $)$ & $\mathrm{I}(\mathrm{err})$ & $\mathrm{H} \alpha-\mathrm{R}$ & $\mathrm{S} / \mathrm{N}$ & $\mathrm{N}$ \\
\hline 187498 & 042017.07 & +324720.8 & $21.23(02)$ & $\overline{20.21(01)}$ & $18.86(01)$ & $-0.34(02)$ & 18.92 & 1 \\
\hline b 108811 & 042130.28 & +330729.2 & $21.05(01)$ & $20.28(01)$ & $19.79(02)$ & $-0.76(01)$ & 41.77 & 2 \\
\hline *a 97731 & 042142.72 & +325427.1 & $21.87(02)$ & $20.80(01)$ & $19.84(02)$ & $-1.45(01)$ & 61.43 & 3 \\
\hline 99419 & 042140.58 & +331204.1 & $21.71(02)$ & $21.14(01)$ & $20.77(05)$ & $-0.45(02)$ & 19.18 & 4 \\
\hline 37863 & 042243.56 & +331332.3 & - & $21.28(03)$ & $19.55(03)$ & $-0.35(04)$ & 8.30 & 5 \\
\hline 226061 & 041953.04 & +330828.0 & - & $21.60(03)$ & $20.77(08)$ & $-0.42(04)$ & 8.56 & 6 \\
\hline 170813 & 042033.62 & +323948.8 & - & $21.91(03)$ & $20.19(03)$ & $-0.32(04)$ & 6.58 & 7 \\
\hline *81814 & 042200.25 & +325708.0 & $23.34(07)$ & $21.98(03)$ & $21.42(08)$ & $-0.32(04)$ & 6.44 & 8 \\
\hline 130822 & 042111.70 & +323838.4 & $22.98(07)$ & $22.18(03)$ & $21.46(09)$ & $-0.55(05)$ & 8.71 & 9 \\
\hline 133739 & 042108.55 & +330810.4 & 23.16(09) & $22.22(04)$ & $21.31(07)$ & $-0.36(06)$ & 5.43 & 10 \\
\hline 165944 & 042037.67 & +330957.1 & $23.16(07)$ & $22.36(03)$ & $21.68(09)$ & $-0.43(05)$ & 6.75 & 11 \\
\hline 197374 & 042007.98 & +324428.8 & $23.17(08)$ & $22.43(04)$ & - & $-1.04(05)$ & 14.71 & 12 \\
\hline 155336 & 042048.59 & +324522.0 & 23.21(09) & $22.68(04)$ & $22.20(16)$ & $-0.97(06)$ & 11.36 & 13 \\
\hline 133393 & 042109.44 & +324059.9 & $23.57(13)$ & $22.81(06)$ & - & $-0.87(07)$ & 8.29 & 14 \\
\hline${ }^{*} 87347$ & 042154.25 & +324735.2 & $24.51(24)$ & $23.21(07)$ & - & $-1.54(08)$ & 10.20 & 15 \\
\hline 81911 & 042200.32 & +324216.6 & $24.11(16)$ & $23.22(07)$ & $22.62(23)$ & $-0.76(10)$ & 5.40 & 16 \\
\hline 156015 & 042047.83 & +325055.6 & - & $23.29(08)$ & $22.77(25)$ & $-0.89(10)$ & 6.22 & 17 \\
\hline 151985 & 042051.72 & +324517.5 & $24.05(20)$ & $23.63(10)$ & - & $-1.80(11)$ & 8.11 & 18 \\
\hline
\end{tabular}

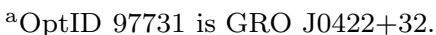

${ }^{\mathrm{b}}$ OptID 108811 is the first CV discovered under the ChaMPlane survey, located outside the Chandra ACIS FoV.

${ }^{*}$ Within the ACIS-I FoV.

It was found outside the ACIS FoV so we do not know its $\mathrm{X}$-ray properties. Its CV status was confirmed via spectroscopy (Rogel et al. 2005). ID 87347 is a very strong $\mathrm{H} \alpha$ emission object with $\mathrm{H} \alpha-\mathrm{R}=-1.54$ and $\mathrm{S} / \mathrm{N}=10.2$, corresponding to $\mathrm{EW}=320 \AA$ based on Figure 4 . It is also (barely) detected in B images taken with the Wide Field Camera on the 2.5-m Isaac Newton Telescope on La Palma, on Jan 13, 2004. A preliminary estimate gives $\mathrm{B}=25.1(2)$. This object is inside the ACIS-I FoV. However, no X-ray emission was detected from this object. The (unabsorbed) flux detection limits $(3-\sigma)$ of this object are $u F x(0.5-2.0 \mathrm{keV}) \leqslant 2.5 \times 10^{-15} \mathrm{ergs} \mathrm{cm}^{-2} \mathrm{sec}^{-1}$, $u F x\left(2.0-8.0 \mathrm{keV}^{-} \leqslant 9.5 \times 10^{-15} \mathrm{ergs} \mathrm{cm}^{-2} \mathrm{sec}^{-1}\right.$, and $u F x(0.5-8.0 \mathrm{keV}) \leqslant 5.6 \times 10^{-15} \mathrm{ergs} \mathrm{cm}^{-2} \mathrm{sec}^{-1}$, assuming $\mathrm{N}_{\mathrm{H}}=1.9 \times 10^{21}$, based on Schlegel et al. (1998), and a power-law spectrum with $\Gamma=1.7$. This yields the absorbed and unabsorbed flux ratios $F_{x}(H c) / F_{r} \leqslant 10.4$ and $u F_{x}(H c) / u F_{r} \leqslant 5.2$. This object can potentially be a $\mathrm{CV}$, because its $\mathrm{EW}$ is much too large to be a dMe star. Deeper Chandra images and optical spectra are required, though this shows the generally comparable depths of the Mosaic and Chandra-ChaMPlane images. Figure 6 shows the $\mathrm{R}$ and $\mathrm{H} \alpha$ images of these three $\mathrm{H} \alpha$ emission sources.

Table 4 is a list of point Mosaic optical sources (i.e. $\mid$ sharpness $\mid<1$ ) found within the match-radii (Eq. [11) of all the level 2 Chandra sources ${ }^{16}$. XPIPE detected 62 point sources on the four ACIS-I chips. One of the 62 is the target - J0422. The other 61 are all newly discovered X-ray sources, based on a search in the SIMBAD Astronomical Database ${ }^{17}$. 37 of these X-ray sources match with 43 point optical counterparts within the $3-\sigma$ search

\footnotetext{
16 See Hong et al. (2005) for definition of levels; for this ObsID level 2 sources are all the valid sources on the ACIS-I chips

17 SIMBAD Astronomical Database is operated at CDS, Strasbourg, France, http://simbad.u-strasbg.fr/cgi-bin/WSimbad.pl
}

radius. Four of the X-ray sources have two possible counterparts each and one X-ray source has three possible counterparts within the $3-\sigma$ error circle.

Table 5 is a list of extended optical counterparts (i.e. sharpness $\geqslant 1$ ) of Chandra sources from the Mosaic catalog. There are $3 \mathrm{X}$-ray sources which match with 3 extended optical counterparts. They are most likely galaxies.

A search of USNO-A2 and GSC2 catalogs yields 6 and 5 optical counterparts, respectively. However, all of them are duplicates, i.e. they are already included in the list of the Mosaic optical counterparts. Therefore, by combining Table 4 and Table 5] there are 40 Chandra sources matching with 46 (point or extended) optical counterparts, which is the complete set of Chandra optical counterparts in the $\mathrm{J} 0422+32$ field.

Table 6] is a list of 22 Chandra sources without optical counterparts, with their optical magnitude limit as measured with the Mosaic photometry.

Table 7 is a list of 5 bright stars in the Henry Draper (HD) Catalog, found within the ACIS-I FoV through a SIMBAD search, with their known spectral type and (for late-type stars only) expected X-ray flux ranges for late-type stars only based on ROSAT observations (Schmitt \& Liefke 2004). None of these 5 stars were detected by the Chandra observation of J0422. The soft band flux detection limit at position of these 5 stars are also listed in the Table.

Figure 7 shows the final stacked deep $R$ image of the field GRO J0422+32, which consists of 10 individual (two sets of 5 dithered) images, with a total exposure time of 2100 seconds. It also shows the ACIS, Chandra sources and their optical counterparts and $\mathrm{H} \alpha$ emission sources overlay. Figure 8 is the same figure but zoomed in around the ACIS-I aimpoint.

Figure 9] shows the J0422+32 field $(\mathrm{H} \alpha-\mathrm{R})$ vs. R 
TABLE 4

GRO J0422+32 Field: Chandra Optical Counterparts (point sources)

\begin{tabular}{|c|c|c|c|c|c|c|c|c|c|c|c|c|c|c|}
\hline & & $\mathrm{RA}(\mathrm{J} 2000)$ & 0) & $\begin{array}{l}r^{\mathrm{b}} \\
\left({ }^{\prime \prime}\right) \\
\end{array}$ & $\begin{array}{r}d^{c} \\
\left({ }^{\prime \prime}\right) \\
\end{array}$ & $\begin{array}{l}\sigma^{\mathrm{d}} \\
(\sigma) \\
\end{array}$ & $\begin{array}{l}F_{x} \\
(\mathrm{Bc})^{\mathrm{e}}\end{array}$ & $\begin{array}{l}F_{x} / F_{r} \\
(\mathrm{Sc})^{\mathrm{f}}\end{array}$ & $\begin{array}{r}F_{x} / F_{r} \\
(\mathrm{Hc})^{\mathrm{g}}\end{array}$ & $\begin{array}{l}V(\text { err }) \\
\text { (mag) }\end{array}$ & $\begin{array}{l}R(\text { err }) \\
\text { (mag) }\end{array}$ & $\begin{array}{l}\text { I(err) } \\
\text { (mag) }\end{array}$ & $\begin{array}{r}\mathrm{H} \alpha-\mathrm{R} \\
(\mathrm{mag}) \\
\end{array}$ & \\
\hline & & & & 0.94 & .20 & 0.63 & 4394 & & & $88(02)$ & $1.53(02)$ & $0.72(04)$ & $0.17(04)$ & \\
\hline & & 21 & & & & & & & & & & & & \\
\hline & & 21 & 257 & & 0.29 & & 62 & & & & 6) & & & \\
\hline & & 21 & 9 & & & & .53 & 17 & & & & & & \\
\hline & & 220 & +325708.0 & 06 & 15 & 1.13 & 2.71 & & 02 & & 3) & 1.4 & 4) & 44 \\
\hline & & 21 & +3258 & 16 & 0.21 & 0.44 & 7.27 & & & & & & & 19 \\
\hline & & 2144 & +330029.2 & 1.52 & 0.35 & 0.70 & 22.68 & & & & & & & 86 \\
\hline & & 221 & 0126.9 & 53 & 0.12 & 0.06 & 25 & & & & & & & 00 \\
\hline & & 220 & +325707.0 & 4.29 & 3.21 & 2.25 & & & & & & & & 42 \\
\hline & & 22 & +32570 & 4.29 & 56 & & & & & & & & & 28 \\
\hline & & 215 & & 3.47 & & 1.24 & 22.22 & & & & & & & 52 \\
\hline & & 215 & & & & 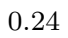 & & & & & & & & 17 \\
\hline & & 22 & & 78 & & & & & & 01 & & & & 04 \\
\hline & & 21 & & 6.77 & & & & & & 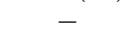 & & & & 31 \\
\hline & & 1 & & 6.77 & & & & & & & & & & .63 \\
\hline & & 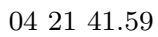 & & 6.77 & & & & & & & & & & 19 \\
\hline & & 01 & & & & 0 . & & & & & & & & \\
\hline & & 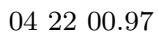 & & & & 73 & & & & & & & & 72 \\
\hline & & 6 & & & 0.20 & & & & & & & & & 03 \\
\hline & & 2 & & & & & & & 20 & & & & & 22 \\
\hline & & 21 & & & & & & & - & & & & & 76 \\
\hline & & 21 . & & & & & & & & & & & & 23 \\
\hline & & Q & & 3.23 & & & & & & & & & & .51 \\
\hline & & & & & & 2.39 & & & & & & & & 64 \\
\hline & & 21 & & & & & & & & & & & & 40 \\
\hline & & 21 & & & & & & & & & & & & .29 \\
\hline & & 0421 & & & & & & & & 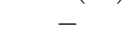 & & & & 05 \\
\hline & & 0421 & +32515 & 0.84 & & & & & & & & & & .14 \\
\hline & 731 & 04214 & +325427 & 0.70 & & 0.4 & 10. & & & & & & & 1.43 \\
\hline & & 0421 : & +325327.3 & 0. & & & & & & 18.3 & & & & 24.40 \\
\hline & & 04214 & & & & & & & & - & & & & 0.95 \\
\hline & & 04212 & +32530 & & & & & & & & & & & 15.82 \\
\hline & & 04212 & +325038 & & 0. & & & & & & & & & 26 \\
\hline & & 04212 & +32513 & 2. & 0. & & & & & & & & & 90 \\
\hline & & 04215 & +32472 & 1. & & & & & & & & & & 49 \\
\hline & & 04215 & +32470 & 7. & & 2. & & & & 19.9 & & & & 55 \\
\hline & & 04215 & +32470 & & & 0.4 & & & & 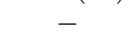 & & & & .23 \\
\hline & & 21 & & & & & & & & & & 2 & 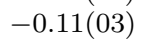 & 22 \\
\hline & & 21 & +324 & & & 1. & & & & & & - & - & .00 \\
\hline & & 0421 & +32 & & 0.6 & 1.2 & & & & & & & & 28 \\
\hline & & 21 & & 3. & & & & & & 17.7 & & 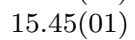 & & 33 \\
\hline & & 21 & +324 & 7. & 7.2 & & & & & - & & - & & 0 \\
\hline 21 & 1522 & 04211 & +324748.2 & 7.30 & 1.63 & 06 & 7.26 & 94 & 1647 & 22.50( & 21. & - & 05) & 0.61 \\
\hline
\end{tabular}

aThe full Chandra SrcID has prefix XS00676 for the J0422+32 observation (ObsID 676).

${ }^{\mathrm{b}} r$ is the $3-\sigma$ match-radius of the X-ray source in arcsec.

${ }^{\mathrm{c}} d$ is the matching distance between X-ray source and its optical counterpart in arcsec.

$\mathrm{d} \sigma$ is the matching distance in unit of $1-\sigma$ error radius.

${ }^{\mathrm{e}} F_{x}(B c)$ is the absorbed broad band $(0.5-8.0 \mathrm{keV})$ flux in unit of $10^{-15} \mathrm{ergs} \mathrm{cm}^{-2} \mathrm{sec}^{-1}$. The unabsorbed- $F_{x}(B c)=1.130 \times F_{x}(B c)$.

${ }^{\mathrm{f}} F_{x}(S c) / F_{r}$ is the ratio of absorbed soft band flux $F_{x}(0.5-2.0 \mathrm{keV})$ vs. observed optical R band flux $\left.F_{r}\left(\operatorname{ergs~cm}^{-2} \sec ^{-1}(1000 \AA)\right)^{-1}\right)$.

The unabsorbed flux-ratio $=1.519 \times F_{x}(S c) / F_{r}$

${ }^{\mathrm{g}} F_{x}(H c) / F_{r}$ is the ratio of absorbed hard band flux $F_{x}(2.0-8.0 \mathrm{keV})$ vs. $F_{r}$. The unabsorbed flux-ratio $=1.017 \times F_{x}(H c) / F_{r}$

${ }^{\mathrm{h}}$ Two Chandra sources have strong $\mathrm{H} \alpha$ emission $(H \alpha-R \leq-0.30 \& S / N \geq 5.0)$ : 97731 is J0422+32; 81814 is a $\mathrm{QSO}$ at $\mathrm{z}=4.25$.

${ }^{\mathrm{i}}$ Three Chandra sources have weak $\mathrm{H} \alpha$ emission $(-0.30<H \alpha-R \leq-0.19$ and $S / N \geq 5.0): 106618$ and 111135 are dMe stars;

111329 is a QSO at $\mathrm{z}=1.31$.

${ }^{*} F_{x}(B c), F_{x}(S c)$ and $F_{x}(H c)$ are calculated using a power-law spectral model with $\Gamma=1.4$.

color-magnitude and (V-R) vs. (R-I) color-color diagrams within the ACIS-I FoV. Figure 10 shows the same diagrams of objects outside the ACIS-I FoV.

\subsection{Follow-up Observations}

The next step of the ChaMPlane optical survey is to obtain the optical spectra of all the $\mathrm{H} \alpha$ emission sources and the Chandra optical counterparts found in the ChaMPlane photometry in order to determine the nature of the objects. This is the final, crucial step for completing the survey. We have been conducting the spectroscopic follow-up using the WIYN 3.5-m (Rogel et al. 2005) and MMT 6.5-m telescopes for the northern ChaMPlane fields and the CTIO 4-m and Magellan 6.5-m tele- 
TABLE 5

Gro J0422+32 Field: Chandra Optical Counterparts (extended sources)

\begin{tabular}{|c|c|c|c|c|c|c|c|c|c|c|c|c|c|c|}
\hline 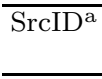 & OptID & $\overline{\mathrm{RA}(\mathrm{J} 2000)}$ & $\overline{\operatorname{Dec}(\mathrm{J} 2000)}$ & $\begin{array}{c}r^{b} \\
\left({ }^{\prime \prime}\right) \\
\end{array}$ & $\begin{array}{r}d^{\mathrm{C}} \\
\left({ }^{\prime \prime}\right) \\
\end{array}$ & $\begin{array}{l}\sigma^{\mathrm{d}} \\
(\sigma) \\
\end{array}$ & $\begin{array}{c}F_{x} \\
(\mathrm{Bc})^{\mathrm{e}}\end{array}$ & $\begin{array}{c}F_{x} / F_{r} \\
(\mathrm{Sc})^{\mathrm{f}}\end{array}$ & $\begin{array}{l}F_{x} / F_{r} \\
(\mathrm{Hc})^{\mathrm{g}}\end{array}$ & $\begin{array}{l}\mathrm{V}(\mathrm{err}) \\
(\mathrm{mag}) \\
\end{array}$ & $\begin{array}{l}\mathrm{R}(\mathrm{err}) \\
(\mathrm{mag}) \\
\end{array}$ & $\begin{array}{l}\text { I(err) } \\
\text { (mag) } \\
\end{array}$ & $\begin{array}{c}\begin{array}{c}\mathrm{H} \alpha-\mathrm{R} \\
(\mathrm{mag})\end{array} \\
\end{array}$ & $\overline{\mathrm{S} / \mathrm{N}}$ \\
\hline$\overline{\mathrm{B} 1 \_005}$ & 71306 & 042212.08 & +325357.4 & 2.45 & 0.36 & 0.45 & 10.01 & - & - & $19.14(04)$ & - & $17.38(04)$ & - & 0.00 \\
\hline B1_009 & 81365 & 042200.85 & +325104.0 & 3.29 & 0.95 & 0.86 & 7.29 & 0.382 & 1.013 & $22.71(06)$ & $21.41(03)$ & $20.31(04)$ & $0.05(05)$ & 1.01 \\
\hline B2_001 & 105760 & 042133.83 & +325557.4 & 0.70 & 0.32 & 1.36 & 31.96 & 1.018 & 4.109 & $21.88(05)$ & $21.05(06)$ & $20.08(05)$ & $0.18(09)$ & 1.89 \\
\hline
\end{tabular}

TABLE 6

GRO J0422+32 Field: Chandra Sources without Optical Counterparts

\begin{tabular}{|c|c|c|c|c|c|c|c|c|c|}
\hline \multirow[b]{2}{*}{$\mathrm{SrcID}^{\mathrm{a}}$} & \multirow[b]{2}{*}{$\mathrm{RA}(\mathrm{J} 2000)$} & \multirow[b]{2}{*}{$\operatorname{Dec}(\mathrm{J} 2000)$} & \multirow[b]{2}{*}{$\begin{array}{c}F_{x} \\
(\mathrm{Bc})^{\mathrm{b}}\end{array}$} & \multirow[b]{2}{*}{$\begin{array}{c}F_{x} \\
(\mathrm{Sc})^{\mathrm{c}}\end{array}$} & \multirow[b]{2}{*}{$\begin{array}{c}F_{x} \\
(\mathrm{Hc})^{\mathrm{d}}\end{array}$} & \multicolumn{4}{|c|}{ Magnitude limits } \\
\hline & & & & & & $\begin{array}{c}\mathrm{V} \\
(\mathrm{mag})\end{array}$ & $\begin{array}{c}\mathrm{R} \\
(\mathrm{mag})\end{array}$ & $\begin{array}{c}\mathrm{I} \\
(\mathrm{mag})\end{array}$ & $\begin{array}{c}\mathrm{H} \\
(\mathrm{mag})\end{array}$ \\
\hline 5 & 7 & 1 & & 1.07 & 3.53 & 24.80 & 24.70 & 22.90 & 4.30 \\
\hline & & & & & & & & & \\
\hline & & & & & & & & & \\
\hline & & & 13 & & 96 & & & & \\
\hline & & & 14.47 & & 51 & & & & \\
\hline & & & & & & & & & \\
\hline & & & & & 2.25 & & & & \\
\hline & & & & & & & & & \\
\hline & & & & & 28 & & & & \\
\hline & & & 9.76 & 2.31 & 33 & & & & \\
\hline & & & 4.01 & - & & & & 84 & 30 \\
\hline & & & 22 & 0.89 & 56 & & & 87 & 16 \\
\hline & & & 18 & 4.71 & 11.26 & & & 96 & 4.43 \\
\hline & & +32 & 4.14 & 0.48 & 5.43 & 24.89 & 24 & 87 & 24.18 \\
\hline & 04211 & +32 & 7.03 & 2.19 & 2.58 & 24.55 & 24.67 & 22.90 & 24.22 \\
\hline & 0421 & +32 & 7.67 & 1.73 & 5.85 & 24.70 & 24.94 & 22.84 & 24.44 \\
\hline & 0421 & +32 & 14.85 & 3.97 & 8.29 & 24.59 & 24.90 & 99 & 24.16 \\
\hline & 04214 & +324944.7 & 8.79 & 2.10 & 6.03 & 24.58 & 25.03 & 23.10 & 24.33 \\
\hline & 042146 & +32 & 1. & 0.50 & 1.14 & 24.67 & 24.73 & 22.85 & 24.20 \\
\hline & 042126. & +324858.9 & 5.06 & 1.45 & 2.31 & 24.61 & 24.88 & 23.04 & 24.09 \\
\hline & 04212 & +32522 & 2.90 & 1.15 & - & 24.76 & 24.84 & 22.97 & 24.40 \\
\hline B3_020 & 042118.66 & +325255.6 & 6.19 & 1.79 & 2.79 & 24.65 & 24.73 & 22.91 & 24.25 \\
\hline
\end{tabular}

${ }^{a}$ The full Chandra SrcID has prefix XS00676 for the J0422+32 observation (ObsID 676). ${ }^{\mathrm{b}} F_{x}(B c)$ is the absorbed broad band $(0.5-8.0 \mathrm{keV})$ flux in unit of $10^{-15} \mathrm{ergs} \mathrm{cm}^{-2} \mathrm{sec}^{-1}$. ${ }^{\mathrm{c}} F_{x}(S c)$ is the absorbed soft band $(0.5-2.0 \mathrm{keV})$ flux in unit of $10^{-15} \mathrm{ergs} \mathrm{cm}^{-2} \mathrm{sec}^{-1}$. ${ }^{\mathrm{d}} F_{x}(H c)$ is the absorbed hard band $(0.5-8.0 \mathrm{keV})$ flux in unit of $10^{-15} \mathrm{ergs} \mathrm{cm}^{-2} \mathrm{sec}^{-1}$. ${ }^{\mathrm{e}}$ Magnitude limit is 5- $\sigma$ above the sky RMS.

TABLE 7

J0422+32 Field: Bright HD Stars Within the ACIS-I FoV

\begin{tabular}{|c|c|c|c|c|c|c|c|c|c|c|}
\hline $\mathrm{ID}^{\mathrm{a}}$ & "RA(J2000) & 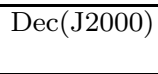 & B & $\overline{\mathrm{V}}$ & $\overline{\text { Type }}$ & $\begin{array}{r}\mathrm{d}^{\mathrm{b}} \\
(\mathrm{pc})\end{array}$ & $\begin{array}{c}\mathrm{N}_{\mathrm{H}} / 10^{22 \mathrm{c}} \\
\mathrm{cm}^{-2}\end{array}$ & $\begin{array}{l}\text { Expected } F_{x} \\
\text { Min }-\operatorname{Max}^{\mathrm{d}}\end{array}$ & $\begin{array}{c}F_{x-\lim } \\
(\mathrm{Sc})^{\mathrm{e}}\end{array}$ & $\begin{array}{l}u F_{x}-\lim \\
(\mathrm{Sc})^{\mathrm{f}}\end{array}$ \\
\hline$\overline{\mathrm{HD} 2}$ & 2.70 & $+32: 54: 39.2$ & 9.5 & $\overline{-}$ & B9 & 748 & 0.189 & - & 1.63 & 2.59 \\
\hline HD281969 & $04: 21$ & $+32: 53$ & 11.7 & 11.0 & A2 & 344 & 0.194 & - & 1.56 & 2.50 \\
\hline HD281970 & $04: 21: 46.14$ & $+32: 57: 31.5$ & 11.6 & 10.9 & $\mathrm{~F} 2$ & 183 & 0.188 & $0.08-134$ & 2.11 & 3.34 \\
\hline HD281973 & $04: 21: 11.25$ & $+32: 49: 48.8$ & 10.07 & 9.55 & F8 & 129 & 0.200 & $0.16-270$ & 3.85 & 6.24 \\
\hline HD281971 & $04: 21: 19.78$ & $+33: 00: 17.4$ & 9.5 & - & K0 & 36 & 0.174 & $2.12-789$ & 2.82 & 4.33 \\
\hline
\end{tabular}

a ID from Henry Draper (HD) catalog.

${ }^{\mathrm{b}} \mathrm{d}$ is the distance in parsec, estimated from $\mathrm{V}, \mathrm{M}_{\mathrm{V}}$ (from spectral type), and $\mathrm{E}(\mathrm{B}-\mathrm{V})$, assuming the object is a main sequence star.

${ }^{c}$ The full column $\mathrm{N}_{\mathrm{H}} / 10^{22}$, based on Schlegel et al. (1998).

${ }^{\mathrm{d}}$ The expected X-ray flux range in the ROSAT band $(0.1-2.4 \mathrm{keV})$, in unit of $10^{-15} \mathrm{ergs} \mathrm{cm}^{-2} \mathrm{sec}^{-1}$, assuming main sequence stars, based on Schmitt \& Liefke (2004). The expected X-ray flux from giants are even lower.

${ }^{\mathrm{e}} F_{x}$-lim is the absorbed soft band $(0.5-2.0 \mathrm{keV})$ flux detection limit $(3-\sigma)$ of this Chandra observation, in unit of $10^{-15} \mathrm{ergs} \mathrm{cm}^{-2} \mathrm{sec}^{-1}$.

${ }^{\mathrm{f}} u F_{x}$-lim is the unabsorbed soft band flux detection limit $(3-\sigma)$. 


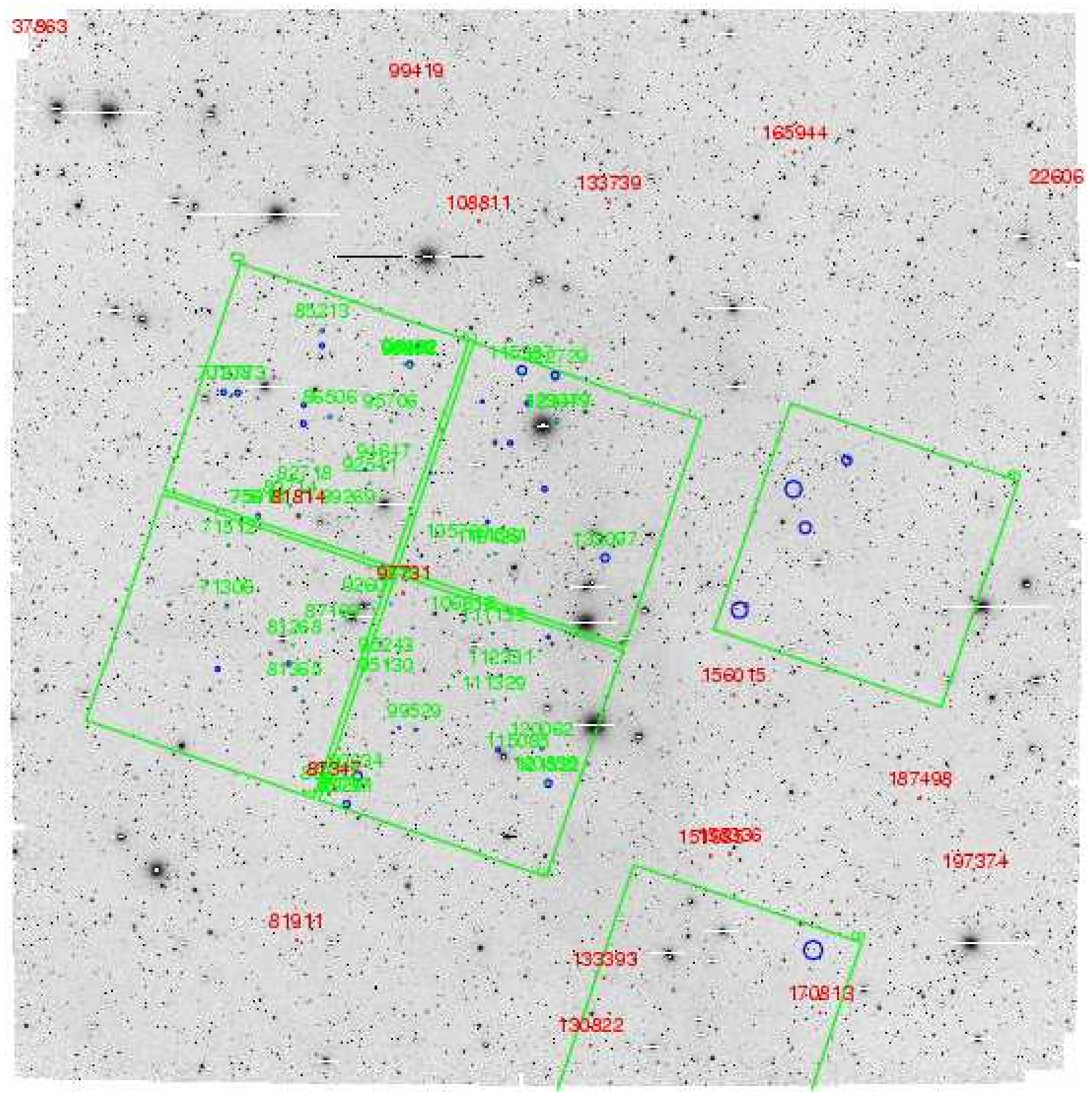

FIG. 7.- GRO J0422+32 field full Mosaic deep R image with active ACIS CCDs overlay (large green squares, ACIS I0-3 and S2,4 were turned on). Chandra source $3-\sigma$ error circles are marked with blue color. Chandra candidate optical counterparts are marked by small ( $1^{\prime \prime}$ ) green circles with their optical ID. $\mathrm{H} \alpha$ emission sources $(\mathrm{H} \alpha-\mathrm{R} \leqslant-0.3$ and $\mathrm{S} / \mathrm{N} \geqslant 5)$ are marked with $\left(1.5^{\prime \prime}\right)$ red circles with their optical ID. ID 108811 (above the ACIS-I) is the first CV discovered under the ChaMPlane project. ID 87347 (near the bottom of ACIS-I) is the previously unknown bright $\mathrm{H} \alpha$ emission object without X-ray detection. Five X-ray sources are detected on the ACIS-S2 and S4 chips. We do not include their optical counterparts here as their positional errors are relatively poorly known due to their large off-axis angles. (North-up, east-left; $36^{\prime} \times 36^{\prime}$ FoV.) (See electronic ApJ for the colored version.) 


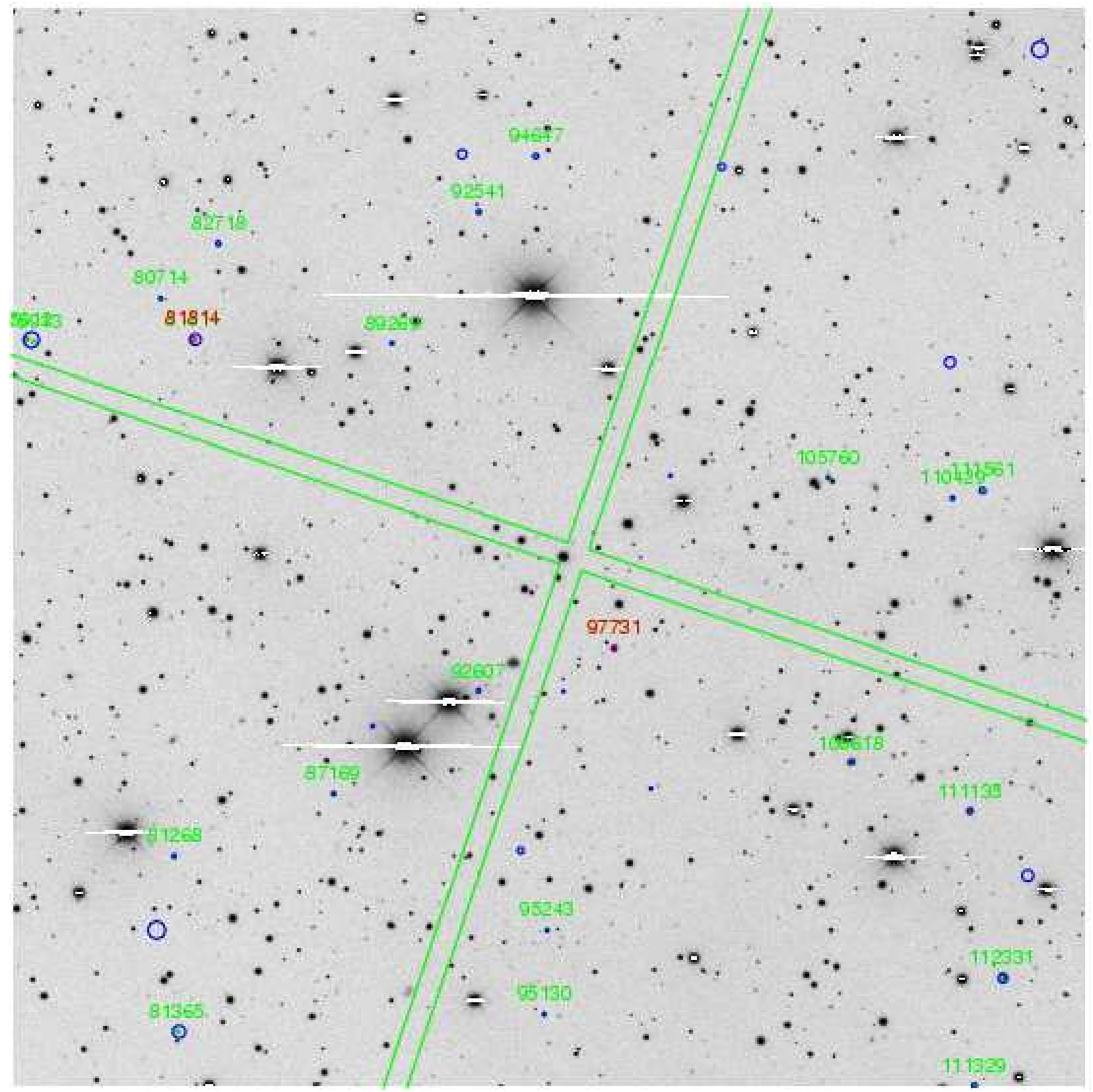

FIG. 8.- GRO J0422+32 field Mosaic deep R image around the ACIS-I aimpoint (See Figure 7 for source marker notations). ID 97731 at the aimpoint is the target J0422+32. ID 81814 is the only other $\mathrm{H} \alpha$ emission Chandra source on ACIS-I, which is a QSO at $\mathrm{z}=4.25$, with Lyman- $\alpha$ red-shifted to Balmer- $\alpha$ (Rogel et al. 2005). (North-up, east-left. ( $9^{\prime} \times 9^{\prime}$ FoV.) (See electronic ApJ for the colored version.)

scopes for the southern fields. Because of the severe extinction in visible bands towards the Galactic center, we are also carrying out infrared imaging photometry for the Galactic bulge fields (Lavcock et al. 2005).

\section{SUMMARY}

We have successfully conducted and completed the optical imaging and Mosaic photometry for the ChaMPlane survey. Considerable followup work (spectroscopy as well as additional photometry analysis) is now in progress to identify the nature of the sources and will be reported in subsequent papers. The photometry survey obtained 65 Mosaic fields, or $\sim 23$ square degrees in the Galactic plane and covers 154 Chandra observations on 105 distinct Chandra fields, which is what we proposed to accomplish. Using 6 Mosaic pointings, we mapped out
2.2 square degrees around the Galactic center using $\mathrm{V}, \mathrm{R}$, I and $\mathrm{H} \alpha$ filters to cover 58 Chandra ACIS observations. This is the deepest optical survey towards the Galactic center, so far.

This paper summarizes our Mosaic photometry observations and describes our data reduction method. Deep Mosaic imaging produces comprehensive optical catalogs for each ChaMPlane field. The $\mathrm{R}$ and $\mathrm{H} \alpha$ differential photometry efficiently detects $\mathrm{H} \alpha$ emission sources. Our search method effectively finds the optical counterparts for the majority of Chandra sources in the low extinction fields. Spectroscopy follow-ups for the Chandra optical counterparts and $\mathrm{H} \alpha$ emission sources for classification complete the ChaMPlane survey. All the optical catalogs produced by the ChaMPlane optical survey will be 

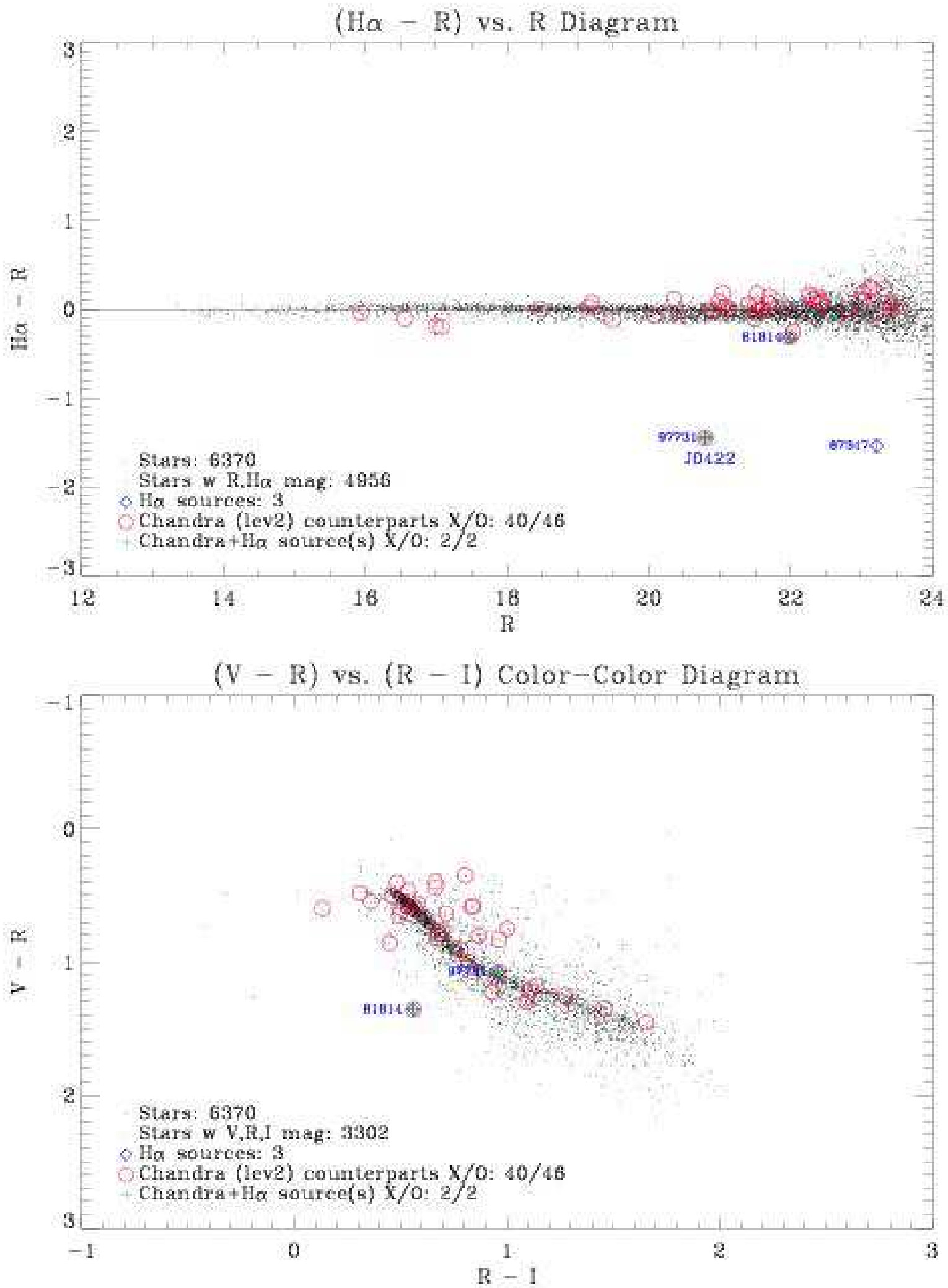

FIG. 9.- GRO J0422+32 field $(\mathrm{H} \alpha-\mathrm{R})$ vs. R color-magnitude and $(\mathrm{V}-\mathrm{R})$ vs. (R-I) color-color diagrams of objects inside ACIS-I FoV. 40 Chandra sources are matched with 46 counterparts (marked as red circles). There are three H $\alpha$ emission sources (marked as blue diamonds) within the ACIS-I FoV. Two of them are Chandra sources: ID 97731 is the black-hole X-ray nova J0422+32 ; ID 81814 is a quasar at $\mathrm{z}=4.25$. ID 87347 is an unclassified bright $\mathrm{H} \alpha$ emission object without X-ray detection. (See electronic ApJ for the colored version.) 

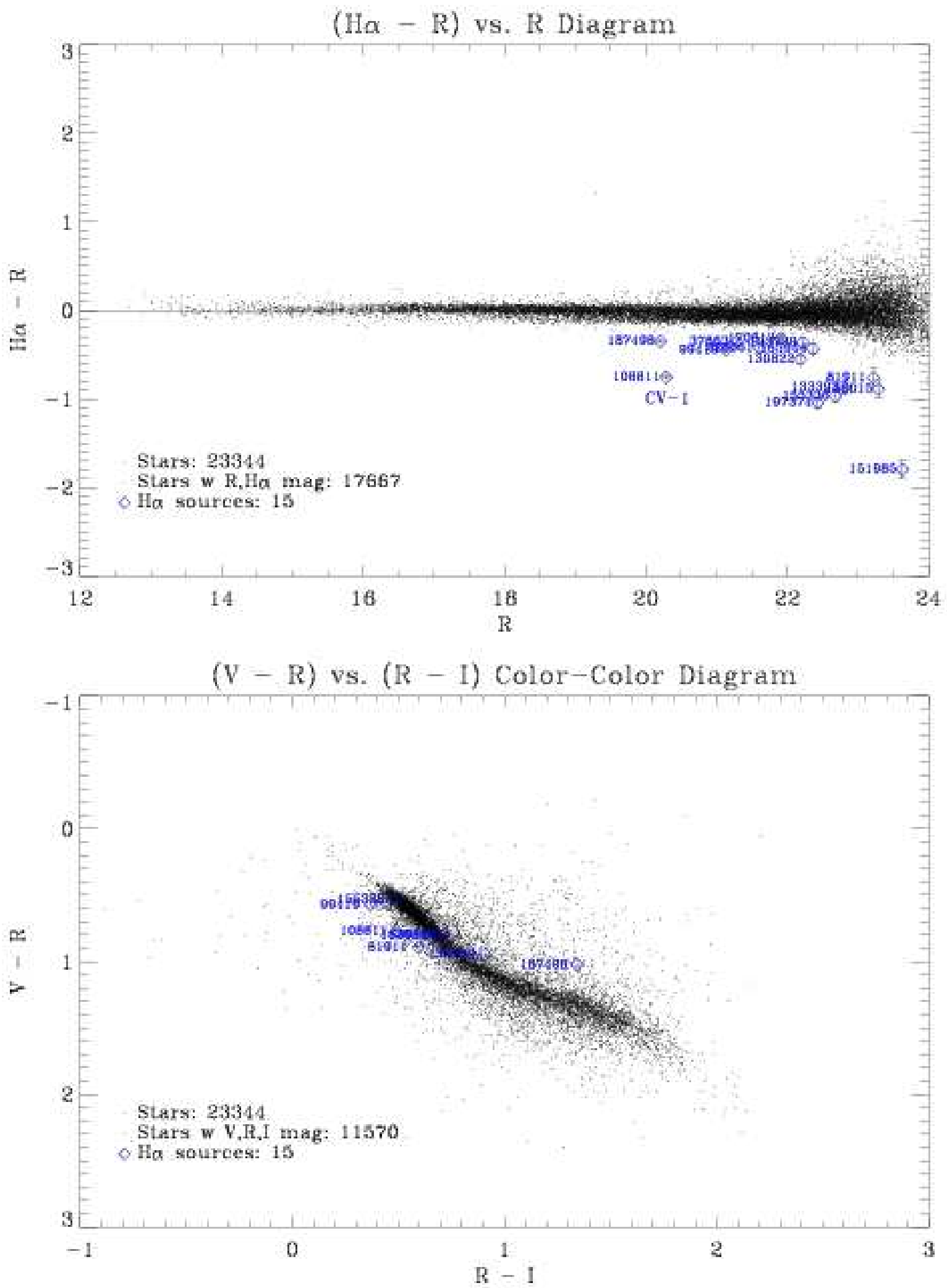

FIG. 10.- GRO J0422+32 field $(\mathrm{H} \alpha-\mathrm{R})$ vs. R color-magnitude and (V-R) vs. (R-I) color-color diagrams of objects outside ACIS-I FoV. There are $15 \mathrm{H} \alpha$ emission sources. The object with ID 108811 is the first CV discovered under the ChaMPlane project. (See electronic ApJ for the colored version.) 
available at the ChaMPlane Online Database and NOAO Science Archive. This legacy Optical Database will provide a rich resource for Galactic astronomy.

We would like to thank following people for participating in varies stages of this project: P.D. Edmonds, J.E. McClintock, M.R. Garcia, R. Cameron, A. Cool, H. Cohn, P. Lugger, A, Rogel, S. Slavin, D. Hoard, and S.
Wachter.

This work is supported in part by NASA/Chandra grants AR1-2001X, AR2-3002A, AR3-4002A, AR45003A, NSF grant AST-0098683 and the Chandra X-ray Center. We thank NOAO for its support via the Long Term Survey program.

Facilities: CXO(ACIS), CTIO 4m(Mosaic), KPNO $4 \mathrm{~m}$ (Mosaic).

\section{REFERENCES}

Grindlay, J.E. et al. 2003, Astronomische Nachrichten, V324, No.1$2,57-60$

Grindlay, J.E. et al. 2005, ApJ, submitted

Hong, J. et al. 2005, ApJ, submitted

Landolt, A.U. 1992, ApJ, 104, 340

Laycock, S. et al. 2005, ApJ, submitted

Mochnacki, S. et al. 2002, AJ, 124, 2868

Muno, M.P. et al. 2003, ApJ 589, 225

Rogel, A.B. et al. 2005, ApJ, submitted

Schlegel, D., Finkbeiner, D., \& Davis, M., ApJ, 1998, 500, 525

Schmitt, J.H.M.M. \& Liefke C. 2004, A\&A 417, 651
Szkody, P. et al. 2002, AJ, 123, 430

Szkody, P. et al. 2003, AJ, 126, 1499

Szkody, P. et al. 2004, AJ, 128, 1882

Szkody, P. et al. 2005, AJ, 129, 2386

Wang, D. et al. 2002, Nature, 415, 148

cf. Warner, B. "Cataclysmic variable stars", Cambridge University Press, 1995

William, G. 1983, ApJS, 53, 523148

Zhao, P. et al. 2003, Astronomische Nachrichten, V324, No.1-2, 176 